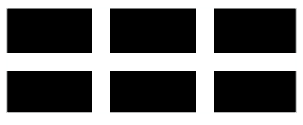

ThE William DAVIDSON INSTITUTE AT THE UNIVERSITY OF MICHIGAN BUSINESS SCHOOL

\title{
Foreign Exchange Interventions in Croatia and Turkey: Should We Give a Damn?
}

\author{
By: Balázs Égert and Maroje Lang
}

William Davidson Institute Working Paper Number 755

March 2005 


\title{
Foreign Exchange Interventions in Croatia and Turkey: Should We Give a Damn?
}

\author{
Balázs Égert* Maroje Lang*
}

\begin{abstract}
This paper studies the impact of daily official foreign exchange interventions on the exchange rates of two EU candidate countries, namely Croatia and Turkey for the periods from 1996 to 2004 and from 2001 to 2004, respectively. Using the event study methodology and a variety of GARCH models reveals that both the Croatian and the Turkish central banks were in a position to influence, to some extent, the level of the exchange rate during the period studied. This lends support to the view that foreign exchange intervention may be effective to a limited extent in emerging market economies.
\end{abstract}

JEL Codes: F31

Keywords: central bank intervention, foreign exchange intervention, official interventions, foreign exchange market, effectiveness, exchange rate volatility, emerging economies, transition economies

\footnotetext{
* Oesterreichische Nationalbank; MODEM, University of Paris X-Nanterre and William Davidson Institute. E-mail: balazs.egert@oenb.at and begert@u-paris10.fr.

-Croatian National Bank, E-mail: mlang@hnb.hr

We would like to thank Evan Kraft, Iikka Korhonen and participants at seminars held at the Croatian National Bank, BOFIT and the Oesterreichische Nationalbank for useful comments and suggestions and Jesús Crespo-Cuaresma for technical help in GARCH programming. Many thanks go to Andreja Pufnik, Danileja Mladinovic, Christoph Fischer and Zoltán Walko for help in obtaining some of the data series used in the paper. Parts of the paper were written while Égert was visiting BOFIT in 2004 and 2005. The opinions expressed in the paper are those of the author and do not necessarily represent the views of the Croatian National Bank, the Oesterreichische Nationalbank or the European System of Central Banks (ESCB).
} 


\section{Introduction}

The question of how, if at all, central bank interventions on the foreign exchange markets may impact on nominal exchange rates has triggered a large body of literature for the last 20 years or so of the post Bretton-Woods period in industrialized OECD economies. Although it is widely acknowledged that unsterilised interventions affect the exchange rate by altering relative money supplies, the empirical evidence is fairly mixed regarding the effectiveness of sterilized interventions, which may work through the portfolio, the signaling and the microstructure (or coordination) channels. In their literature survey, Sarno and Taylor (2001) conclude, however, that what emerges from studies focusing on the 1990s is that interventions tend to impact on the exchange rates, "especially if the intervention is publicly announced and concerted and provided it is consistent with the underlying stance of monetary and fiscal policy"2.

It is a well-documented fact that a large number of emerging market economies have moved recently from fixed exchange regimes towards more flexible exchange regimes. Nevertheless, extensive foreign exchange interventions were undertaken in these countries mainly driven by fear of floating (Calvo and Reinhard, 2000) or by a dread of depreciation, as suggested by Dutta and Leon (2002). In this context, Canales-Kirjenko (2003) puts forth that foreign exchange interventions may be more effective in emerging market economies as compared to wellestablished industrialized countries because of the following reasons: (a) central bank interventions are not always fully sterilized, (b) the size of interventions is large relative to market turnover in narrow forex markets, (c) the market organization and the regulatory framework may be more conducive to interventions, (d) moral suasion may play a bigger role, and (e) because of the larger informational advantage of the central banks vis-à-vis market participants.

\footnotetext{
${ }^{2}$ For limited effectiveness of official interventions, see e.g. Aguilar and Nydahl (2000) for Sweden, Morana and Beltratti (2000) for the USD/DEM, Brandner et al. (2001), Brandner and Grech (2002) for the ERM currencies. Brissimis and Chionis (2004) suggest that interventions by the ECB were not effective for the yen/euro exchange rate. Fatum (2000) finds evidence for effectiveness for the same currency pair. Ramaswamy and Samiei (2000), Fatum and Hutchison (2003) and Brissimis and Chionis (2004) show that sterilised interventions were effective for the yen/USD and yen/euro exchange rates. For mixed evidence for Australia, see e.g. Kim et al (2000), Kearns and Rigobon (2002), Edison et al. (2003) and Rogers and Siklos (2003).
} 
However, there is little empirical research conducted to investigate the effectiveness of central bank interventions in emerging and transition economies. ${ }^{3}$ In this paper, daily intervention data are used to study the extent to which foreign exchange interventions were effective in Croatia from 1996 to 2004 and in Turkey from 2001 to $2004 .{ }^{4}$ In a first step, we apply the event study approach, which is claimed to be superior to econometric analysis if interventions take place only sporadically (Fatum, 2000 and Fatum and Hutchison, 2003), and analyze the influence of official interventions on the mean and the variance of the exchange rates vis-à-vis the German mark prior to 1999 and vis-à-vis the euro after 1999 for Croatia, and vis-à-vis the US dollar for Turkey. In a second step, a variety of GARCH models are employed to get an econometric grip on the data.

The remainder of the study is structured as follows. Section 2 sketches briefly monetary and exchange rate policies and foreign exchange interventions for Croatia and Turkey for the periods 1996 to 2004 and 2001 to 2004, respectively. Section 3 presents the results of the event study approach. Section 4 contains the estimation results of the different GARCH specifications and the causality tests. Section 5 finally gives some concluding remarks.

\section{Exchange Rate Regimes and Foreign Exchange Interventions}

\subsection{Croatia}

Croatia has been having a managed float exchange rate regime since the introduction of the kuna in April 1994. Exchange rate policy has been ever since oriented towards the German mark and subsequently towards the euro even if there was no official currency basket. During the period from January 1996 to November 2004, the exchange rate of the kuna was kept in a fluctuation band of $-6.7 \% /+5.2 \%$ relative to the period average and the implicit fluctuation band was even narrower during some periods of time as can be seen from Figure 1.

Nominal exchange rate targeting, which is publicly communicated by the Croatian National Bank (see e.g. Croatian National Bank, 2001, 2002 and 2003), has its main goal to achieve price

${ }^{3}$ Canales-Kriljenko (2003), Canales-Kriljenko et al. (2003) and Mihaljek (2005) provides an overview on foreign exchange intervention practices in emerging market economies. Domac and Mendoza (2004) and Guiamaraes and Karacadog (2004) study daily foreign exchange interventions for the cases of Turkey and Mexico. 
stability. Back in 1994, the managed floating regime and the new kuna were introduced to stem hyperinflation as high as $1518 \%$ on average in 1993. Yearly average inflation rapidly went down to $2.0 \%$ in 1995 and has been ever since in low single-digit territories (1.8\% in 2003). The nominal exchange rate target secures price stability through stable imported inflation (and via its second round effects), an important factor in a highly euroised country such as Croatia, and, perhaps more importantly, it acts as an anchor for inflation expectations.

The Croatian National Bank (CNB) regularly intervenes on the foreign exchange market to stabilise the kuna against the euro (German mark) in both directions. Excessive exchange rate movements are actually interpreted by the CNB as an appreciation or a depreciation of the kuna vis-à-vis the euro of about $2 \%$ and higher than $2 \%^{5}$. It should be noted that part of the exchange rate movements is due to seasonal factors. The kuna tends to depreciate at the beginning and at the end of the year because of a seasonal worsening of the current account, and it appreciates during the summer period because of an increased demand for kunas in the tourist season. If judged excessive, the central bank also acts to counteract seasonal movements in the exchange rate.

Foreign exchange interventions are achieved through foreign exchange auctions, where the central bank sells or buys domestic currency to and from domestic commercial banks. From 2001 onwards, off-market interventions also took place to prevent appreciation pressures coming from the conversion of privatization revenues (Croatian Telecom, 2001; INA (Croatian oil company), 2003). The central bank bought or sold foreign currency to and from the Ministry of Finance and the Ministry of Finance held the foreign exchange obtained from issuing eurobonds on its account at the CNB.

Foreign exchange interventions are sterilized by the central bank mainly (a) via reserve requirements for the banks and (b) by issuing foreign currency and kuna central bank bills. The CNB has recently made efforts to move towards more market-based instruments. In this attempt,

\footnotetext{
${ }^{4}$ Other CEECs are not included because of the lack of daily intervention data.

${ }^{5}$ CNB (2001,p. 33): «The kuna/euro value grew by $1.8 \%$ in nominal terms in July in comparison with end-June, while it had strengthened by $0.5 \%$ in July 2000 . In an effort to eases the intense appreciation of the exchange rate of the kuna against the euro, the central bank purchases from banks a total of USD $122.9 \mathrm{~m} »$.

CNB (2001,p. 34) : «The last quarter of 2001 was marked by a nominal appreciation of the kuna against the euro of $2.3 \%$ (...). In an effort to keep exchange rate movements within satisfactory stability boundaries, the central bank purchased from banks a total of USD $338.5 \mathrm{~m} »$

CNB (2003,p 40) : «In this period, the kuna weakened against the euro by a total of $3.4 \%$ (...). The kuna depreciation stimulated the sale of foreign exchange at the central bank's auctions in the first quarter of 2003. »
} 
it unified the kuna and foreign currency requirements in 2000 (e.g. regarding the calculation period and the maintenance period). Since 2001, the reserve requirement rate was decreased several times, and this was compensated by a widening of the calculation base (CNB, 2001, 2002, and 2003).

Figure 1 and Table 1 show foreign exchange interventions via auction in the interbank foreign exchange market. The Croatian National Bank intervened on 187 occasions from 1996 to 2004 : It purchased the domestic currency on 88 occasions and sold it 99 times. The CNB's intervention activity was intensive in 1996, 1998 and 1999 with over 30 yearly interventions. 2001 and 2002 are characterized by roughly 20 interventions a year, while 1997 and more recently 2003 and 2004 can be viewed as periods of calm with about 10 interventions a year. Although the number of sales and purchases is fairly balanced for the period as a whole, sales outweighed purchases in 1996, 1997, 2000, 2001, 2002 and 2004, whilst purchases occurred more frequently in 1998, 1999 and 2003.

Figure 1. Interventions and the kuna/euro (German mark) exchange rate, Croatia, 1996-2004

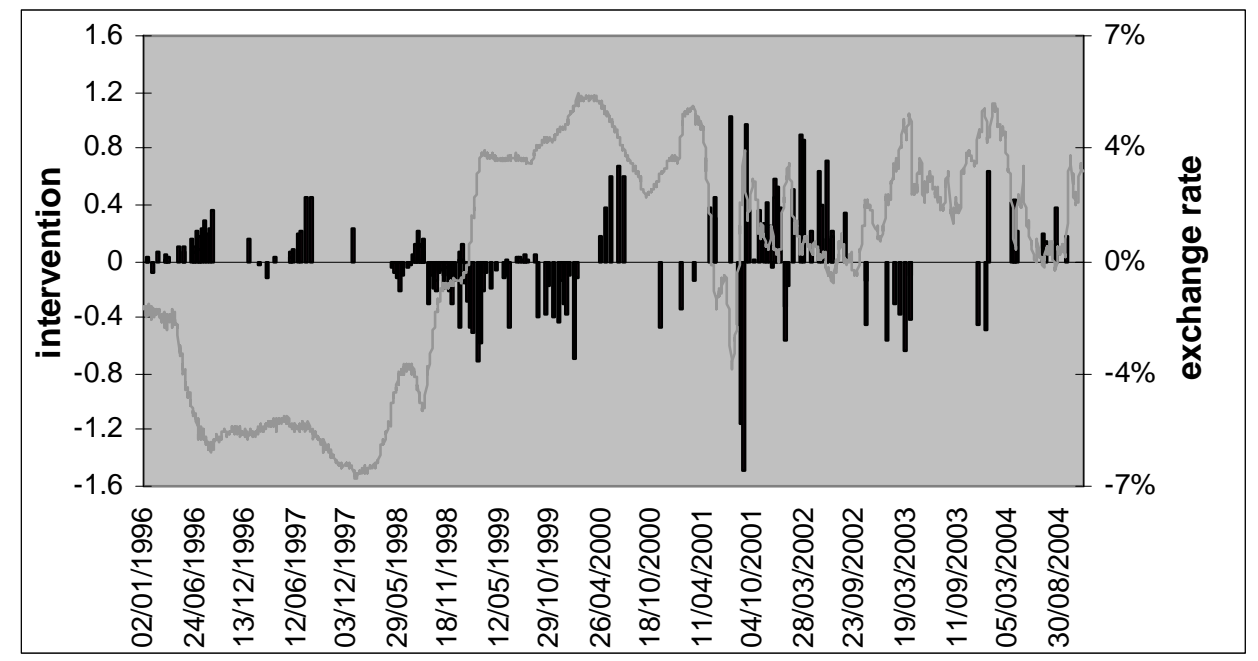

Source: Croatian National Bank

Notes: Interventions are in billions of Croatian kuna. Negative (positive) values show kuna purchases (sales). The exchange rate is shown as the deviation from the period average kuna/euro (German mark) exchange rate 
Table 1. Summary of interventions activities by the Croatian National Bank, 1996-2004

(HRK millions)

\begin{tabular}{|c|c|c|c|c|c|c|c|}
\hline \multicolumn{2}{|c|}{ Summary } & Mean & Median & Min & Max & SD & Days of intervention \\
\hline \multicolumn{2}{|c|}{1996 Total } & 73 & 23 & 1 & 369 & 96 & 39 \\
\hline & Sales & 124 & 98 & 4 & 369 & 100 & 22 \\
\hline & Purchases & 7 & 2 & 1 & 76 & 18 & 17 \\
\hline \multirow[t]{3}{*}{1997} & Total & 160 & 105 & 18 & 454 & 155 & 12 \\
\hline & Sales & 195 & 194 & 30 & 454 & 163 & 9 \\
\hline & Purchases & 57 & 32 & 18 & 120 & 56 & 3 \\
\hline \multirow[t]{3}{*}{1998} & Total & 109 & 85 & 1 & 475 & 96 & 43 \\
\hline & Sales & 67 & 58 & 1 & 219 & 59 & 19 \\
\hline & Purchases & 142 & 105 & 31 & 475 & 108 & 24 \\
\hline \multirow[t]{3}{*}{1999} & Total & 229 & 168 & 4 & 716 & 200 & 30 \\
\hline & Sales & 23 & 22 & 4 & 48 & 16 & 8 \\
\hline & Purchases & 304 & 299 & 59 & 716 & 182 & 22 \\
\hline \multirow[t]{3}{*}{2000} & Total & 467 & 533 & 112 & 702 & 225 & 8 \\
\hline & Sales & 490 & 593 & 180 & 684 & 206 & 5 \\
\hline & Purchases & 429 & 472 & 112 & 702 & 298 & 3 \\
\hline \multirow[t]{3}{*}{2001} & Total & 500 & 396 & 14 & 1488 & 378 & 20 \\
\hline & Sales & 455 & 396 & 14 & 1029 & 268 & 14 \\
\hline & Purchases & 606 & 397 & 54 & 1488 & 580 & 6 \\
\hline \multirow[t]{3}{*}{2002} & Total & 421 & 374 & 137 & 904 & 245 & 17 \\
\hline & Sales & 458 & 386 & 152 & 904 & 265 & 12 \\
\hline & Purchases & 332 & 326 & 137 & 564 & 183 & 5 \\
\hline \multirow[t]{3}{*}{2003} & Total & 444 & 458 & 99 & 646 & 171 & 9 \\
\hline & Sales & 646 & 646 & 646 & 646 & 0 & 1 \\
\hline & Purchases & 418 & 439 & 99 & 635 & 164 & 8 \\
\hline \multirow[t]{3}{*}{2004} & Total & 250 & 199 & 129 & 440 & 126 & 9 \\
\hline & Sales & 250 & 199 & 129 & 440 & 126 & 9 \\
\hline & Purchases & -- & -- & -- & -- & -- & 0 \\
\hline \multirow[t]{3}{*}{ 1996-2004 } & Total & 232 & 152 & 1 & 1488 & 245 & 187 \\
\hline & Sales & 234 & 163 & 1 & 1029 & 236 & 99 \\
\hline & Purchases & 231 & 138 & 1 & 1488 & 256 & 88 \\
\hline
\end{tabular}

\subsection{Turkey}

Although Turkey's exchange rate stabilization program, which rested on a pre-announced crawling peg exchange rate regime, had the merit to having brought down year-on-year inflation from 68.8\% in December 1999 to 39\% in December 2000, it culminated in a currency crisis in early 2001. As a result, a floating exchange rate regime was introduced on February 22, 2001. This was part of a new monetary policy, which can be best described as an implicit inflation targeting. Under this regime, the central bank pursues an inflation target at a given horizon in the future. The central bank's main instruments are short-term interest rates. The base money and net international reserves are used as "indicative criteria", which can be perhaps deemed as intermediate targets. Net domestic assets, measuring domestic credit expansion, are considered as an indicator for monetary policy. This monetary policy framework was underpinned with the amendment of the Central Bank Act on May 5, 2001, aimed at securing the operational independence of the central bank. 
The role of foreign exchange interventions is understood to be limited in such a framework. The Central Bank of the Republic of Turkey (CBRT) has been stressing in its official publications that foreign exchange interventions should take place as rarely as possible and in a transparent manner (CBRT, 2001, p. 109 and CBRT, 2004, paragraph 34). In addition to this, it is also emphasized that foreign exchange interventions do not intend to target any precise level of the exchange rate but aims to dampen excessive volatility instead and is used for building international reserves. ${ }^{6}$ When considering exchange rate volatility, the central bank not only looks at past and present volatilities but also considers expected changes in volatility occurring in the future (CBRT, 2004, paragraph 26).

However, reading between the lines may reveal that the central bank may give a lower weight to decreasing excessive exchange rate volatility and it may ponder to alter the trend of the exchange rate. According to CBRT (2002, p. 74), "foreign exchange auctions were temporarily suspended as of July due to the volatilities of exchange rates", which is in contradiction with the declared intention to counteract excessive volatility. It is also mentioned several times that the central bank considers excessive volatility in both directions. Given that volatility is an absolute measure, this may indicate that the CBRT also looks at changes in the exchange rate. ${ }^{7}$

The central bank carries out several operations to sterilize foreign exchange interventions by means of (1) Turkish lira deposit operations in the interbank money market and (2) reverse repo transactions at the Istanbul Stock Exchange. It also ensures that short-term money market interest rates remain in line with the inflation target.

The interventions of the central bank were mostly carried out based on either discretionary or pre-announced auctions. However, the central bank also did some direct discretionary interventions on the forex markets. The amount of these discretionary interventions is not

\footnotetext{
${ }^{6}$ CTB (2001, p. 109) : « The Central Bank conducted regular FX sales auctions after March 29 in order to smooth excessive short-run exchange rate fluctuations without affecting the long-run equilibrium level of exchange rates... »

CTB (2002, p. 71) : " ...the Central Bank announced that it would intervene in the foreign exchange rate market in a strictly limited fashion to prevent excessive volatility without targeting a certain trend level. »

CTB (2004, paragraph 34): «The Bank has not performed any foreign exchange buying or selling operations intended at determining the level or direction of the exchange rates. The Bank's foreign exchange buying or selling operations aimed at controlling excessive volatilities. »

${ }^{7} \mathrm{CTB}(2003, \mathrm{p} .97)$ : « (...) it would directly intervene in the event of excessive volatility that might occur in both directions ». CTB (2002, p. 96) : « (...) the Central Bank did not target any exchange rate level and that it would respond symmetrically to both upward and downward volatility. »
} 
reported by the central bank. ${ }^{8}$ Table 3 and Figure 2 provide an overview of the interventions via discretionary and pre-announced auctions, from which it can be seen that the central bank intervened massively on four occasions. First, it purchased Turkish lira 124 times in 2001 in the aftermath of the crisis. The remaining three intervention episodes are all lira selling operations, which contain 33 (2002), 117 (2003) and 62 (2004) separate intervention acts. As reported in diverse central bank publications, these operations resulted in a substantial increase in international reserves, which is consistent with the monetary policy framework (international reserves are an intermediate target).

Figure 2. Interventions and the Turkish lira/dollar exchange rate, Turkey, 2001-2004

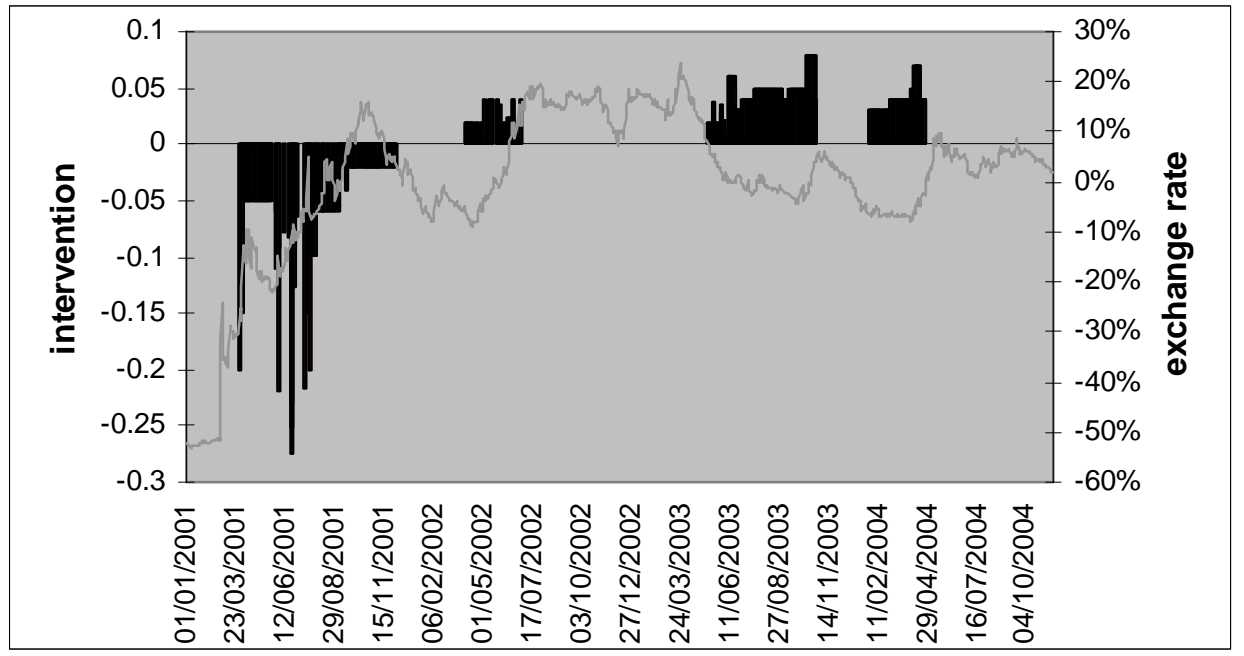

Source: Central Bank of the Republic of Turkey

Note: Interventions are in billions of USD. Negative (positive) values show lira purchases (sales). The exchange rate is shown as the deviation from the period average lira/dollar exchange rate

Table 2. Summary of interventions activities by the Central Bank of the Republic of Turkey 2001-2004 (USD millions)

\begin{tabular}{|c|c|c|c|c|c|c|c|}
\hline Summary & & Mean & Median & Min & Max & SD & Days of intervention \\
\hline 2001 & Total & 53 & 50 & 20 & 274 & 51 & 124 \\
\hline & Sales & -- & -- & -- & -- & -- & 0 \\
\hline & Purchases & 53 & 50 & 20 & 274 & 51 & 124 \\
\hline 2002 & Total & 24 & 20 & 4 & 40 & 10 & 33 \\
\hline & Sales & 24 & 20 & 4 & 40 & 10 & 33 \\
\hline & Purchases & -- & -- & -- & -- & -- & 0 \\
\hline 2003 & Total & 43 & 50 & 2 & 80 & 18 & 117 \\
\hline & Sales & 43 & 50 & 2 & 80 & 18 & 117 \\
\hline & Purchases & -- & -- & -- & -- & -- & 0 \\
\hline 2004 & Total & 40 & 40 & 30 & 70 & 11 & 62 \\
\hline & Sales & 40 & 40 & 30 & 70 & 11 & 62 \\
\hline & Purchases & -- & -- & -- & -- & -- & 0 \\
\hline 2001-2004 & Total & 44 & 40 & 2 & 274 & 34 & 336 \\
\hline & Sales & 39 & 40 & 2 & 80 & 16 & 212 \\
\hline & Purchases & 53 & 50 & 20 & 274 & 51 & 124 \\
\hline
\end{tabular}

Source: Author's calculations

\footnotetext{
${ }^{8}$ Guimaraes and Karacadag (2004) provide a useful overview regarding the types of central bank interventions from February
} 2002 to November 2003. 


\section{The Effectiveness of Interventions: An Event Study Approach}

\subsection{Methodological Issues}

If foreign exchange interventions occur rarely or only after a large number of days without intervention, standard time series econometric techniques are likely to find that official interventions do not have an effect on exchange rates, with the latter being highly volatile in the short run (Fatum, 2000; Fatum and Hutchinson,2003). A big advantage of the event study approach over time series techniques is that it only looks at periods when interventions take place, and is thus able to filter out longer periods during which no interventions happen and which might cause econometric studies to find no relation between foreign exchange interventions and exchange rate behavior.

When applying the event study approach, three issues have to be tackled:

(a) how single interventions in one direction can form a single intervention episode or event,

(b) under what circumstances an intervention episode can be viewed as effective/successful.

(c) how long a time horizon should be analyzed before and after the intervention event (definition of pre- and post-event windows)

Regarding the definition of an intervention event, the question is of how many days may separate two single intervention acts going in the same direction (both purchases or both sales) can be considered as two distinct intervention events. If too few, say one or two, days, are taken as the boundary between two intervention events, overlaps between the pre- and post-event may occur, which makes it difficult to interpret the effect of a given intervention event on the exchange rate in the pre- and post event windows because of the simultaneous impact of the overlapping intervention events. Furthermore, setting the distance too short may lead to the overestimation of the intervention episodes. By contrast, if too large a distance is set between single interventions to form a common intervention episode, the risk then is to underestimate the number of true intervention episodes (Fatum, 2000).

Five alternative definitions of the intervention event are considered in this study. We consider intervention events, which comprise single interventions in one direction between which up to 2 , 
5, 10, 20 and 30 consecutive days can pass without intervention activity. The intervention event ends if more than 2, 5, 10, 20 or 30 days go by without intervention or if an intervention in the other direction takes place. ${ }^{9}$

Turning now to measuring the effectiveness of an intervention event, a central bank intervention can be thought of as being effective, if purchases (sales) of the domestic currency result in an appreciation (deprecation). Nevertheless, the definition of success crucially hinges on the direction of the exchange rate prior to the intervention event. In this sense, the following three classes of effective interventions can be distinguished.

Leaning against the wind: the central bank intervention may intend to reverse the trend of the exchange rate. Such an intervention is deemed a success if the exchange rate depreciate (appreciate) in the pre-event window, and following the purchases (sales) of domestic currency, it appreciates (depreciates) in the post-event window:

Buying the domestic currency: $\quad\left(I_{t}<0, \Delta e_{t-}>0\right.$ and $\left.\Delta e_{t_{+}}<0\right)$

Selling the domestic currency: $\quad\left(I_{t}>0, \Delta e_{t-}<0\right.$ and $\left.\Delta e_{t^{+}}>0\right)$

Where $I_{t}<0\left(I_{t}>0\right)$ stands for purchases (sales) of the domestic currency, $\Delta e_{t-}$ is the change in the exchange rate in the pre-event window, and $\Delta e_{t+}$ is the change in the exchange rate in the post-event window.

Smoothing exchange rate movements: the central bank may want to slow down the appreciation or the depreciation of the domestic currency. Such an operation is viewed successful in the event that buying (selling) the domestic currency causes the exchange rate to depreciate less (appreciate more) in the post-event window than in the pre-event window.

Domestic currency purchases: $\quad\left(I_{t}<0, \Delta e_{t^{-}}>0, \Delta e_{t_{+}}>0\right.$ and $\left.\Delta e_{t_{+}}<\Delta e_{t^{-}}\right)$

Domestic currency sales: $\quad\left(I_{t}<0, \Delta e_{t-}<0, \Delta e_{t+}<0\right.$ and $\left.\Delta e_{t_{+}}>\Delta e_{t_{-}}\right)$

Leaning with the wind: Such a strategy involves an amplification of an ongoing trend in the exchange rate market. In such a case, purchases (sales) of the domestic currency should cause the exchange rate to appreciate more (depreciate more) after the intervention episode than before the intervention episode.

\footnotetext{
9 Fatum (2000) and Fatum and Hutchison (2003) use up to 15 days and Edison et al. (2003) use up to 10 days with no intervention between two neighbouring interventions within an event.
} 
Buying the domestic currency: $\quad\left(I_{t}<0, \Delta e_{t-}<0, \Delta e_{t+}<0\right.$ and $\left.\Delta e_{t+}<\Delta e_{t^{-}}\right)$

Selling the domestic currency: $\quad\left(I_{t}>0, \Delta e_{t_{-}}>0, \Delta e_{t+}>0\right.$ and $\left.\Delta e_{t^{+}}>\Delta e_{t^{-}}\right)$

As to the size of the pre- and post-event windows, we look at six different lengths: 2, 5, 10, 20, 30, 40 and 60. The pre- and post-event windows are constructed in a symmetric way implying that a 2-day (5-day etc.) pre-event window is compared to a 2-day (5-day etc.) post-event window. ${ }^{10}$ In addition, effectiveness is also analyzed for the event window itself. The pre-event window is set to $2,5,10,20$ and 30 days if the event window size is equal or lower than 2 days (higher than $2(5,10,20$ and 30) but equal or lower than $5(10,20$ and 30)).

Finally, not only changes in the exchange rate but also changes in the volatility of the exchange rate can be analyzed. For this purpose, volatility measured as standard deviation over the (symmetric) pre- and post-event windows are compared.

\subsection{Results}

\section{Croatia}

Table 3 below documents that in Croatia, the identification of intervention episodes is fairly sensitive to the maximum days of no intervention elapsed between two single intervention acts. Using maximum two days yields a total of 148 intervention episodes, and this number drops to 71 when 10 days are employed. When the criterion is set at 20 or 30 days, the number of identified episodes drops further to 49 and 46, respectively.

Table 3. The number of the identified intervention episodes

\begin{tabular}{|l|l|c|c|c|c|c|}
\hline & & \multicolumn{5}{|c|}{$\begin{array}{c}\text { Maximum days of intervention inactivity between two } \\
\text { consecutive interventions }\end{array}$} \\
\hline & & & \multicolumn{5}{|c|}{ days } & 5 days & 10 days & 20 days & 30 days \\
\hline Croatia & $1996: 01$ to 2004:10 & 148 & 95 & 71 & 49 & 46 \\
\hline Turkey & $2001: 01$ to 2004:06 & 10 & 5 & 4 & 4 & 4 \\
\hline
\end{tabular}

Summary statistics are provided for each filter in Tables 4 and 5. Despite the difference regarding the number of identified intervention episodes, a number of common features can be observed for the intervention episodes based on different filters. First, Table 4 documents the high number of episodes, for which the effectiveness of interventions can be addressed only for

\footnotetext{
${ }^{10}$ Fatum (2000) employs 2, 5, 10, and 15-day window sizes, whilst Edison et al. (2003) looks at 2-day and 21-day windows. Edison and others term the 2-day window the short-term and the 21-day window the long-term.
} 
the pre-event window and the event window itself, but not for the post-event window because the next intervention episode starts the next day or one day later after the episodes considered. This is because the central bank changed the direction of the intervention, and after buying (selling) the domestic currency against the euro (German mark) on one day, it started selling (buying) the kuna the next day or so. In addition to this, for another bunch of episodes, the impact of the episode on changes in the exchange rate can be assessed only at the shorter horizons because of the short distance separating the episodes. The shorter the maximum days of no interventions used for the episode selection, the lower the number of non-overlapping longer pre- and postevent windows. Using maximum 2 days, $46 \%$ of the episodes can be assessed for the pre- and post-event window size of 5 days, and the share of assessable episodes for 10, 20 and 30-day post-event windows drops to $20 \%, 7 \%$ and $3 \%$, respectively. For the post-event window of 60 days, all the episodes overlap with other episodes. Although the share of episodes with no overlap increases with the rise in the maximum days of no intervention, it is still fairly low. For maximum 30 days, for the 10-day post-event window, 35\% of the episodes can be analyzed without overlaps occurring, and the share goes down to $11 \%$ for the 60 -day post-event window. Hence, the effectiveness of central bank interventions can be studied only partially for Croatia using the study event approach.

An episode is qualified as a success if the episode can be viewed as leaning against the wind (WIND), smoothing exchange rate movements (SMOOTH), or leaning with the wind (WITH) in accordance with the criteria explained in the previous section. Table 4 also reports successful and unsuccessful episodes as a share of non-overlapping episodes. The share of successful episodes is fairly high and stable for different post-window sizes and event sizes. It ranges from about $60 \%$ to $80 \%$.

As far a the relative share of the three types of successful intervention (leaning with and against the wind and smoothing) is concerned, Table 4 reveals that the successful intervention the overwhelming majority of episodes classifies as either a leaning against the wind or exchange rate smoothing both within the event window or when comparing the pre- and post-event periods. It appears that exchange rate smoothing is more dominant within the event window, but the share of leaning against the wind usually increases for pre- and post-event windows of 20, 30 and 60 days. This implies that interventions first start to decrease the pace of depreciation or 
appreciation, and as time passes, they even manage to change the trend on the foreign exchange market.

In Croatia, the decision regarding intervention is taken in the morning, and the auction (intervention) is held on the very same day, while the settlement of the intervention occurs two days later. This two-day window, which comprises the announcement of the intervention but which comprises no actual transaction, enables us to study the signaling effect of interventions. In Table 4, under "SIGNAL" are compared changes in the exchange rate prior to a 2-, 5- and 10day pre-event window as compared to exchange rate developments in the two-day window. Table 4 indicates that there is a signaling effect, which is the most pronounced in the very short term (two-day pre-event window).

Table 4. Non-overlapping episodes, and the share of successful episodes, Croatia

\begin{tabular}{|c|c|c|c|c|c|c|c|c|c|c|c|}
\hline \multirow[b]{2}{*}{ EVENT SIZE } & \multicolumn{3}{|c|}{ SIGNAL } & \multirow[t]{2}{*}{$\mathbf{W}$} & \multicolumn{7}{|c|}{ PRE-AND POST-EVENT WINDOW } \\
\hline & 2 & 10 & 20 & & 2 & 5 & 10 & 20 & 30 & 40 & 60 \\
\hline \multicolumn{12}{|l|}{2 DAYS } \\
\hline TOTAL EPISODES & 148 & 148 & 148 & 148 & 148 & 148 & 148 & 148 & 148 & 148 & 148 \\
\hline ASSESSABLE (\% of total) & $97 \%$ & $36 \%$ & $16 \%$ & $100 \%$ & $92 \%$ & $46 \%$ & $20 \%$ & $7 \%$ & $3 \%$ & $2 \%$ & $1 \%$ \\
\hline SUCCESS (\% of assessable) & $59 \%$ & $66 \%$ & $42 \%$ & $66 \%$ & $56 \%$ & $71 \%$ & $80 \%$ & $82 \%$ & $75 \%$ & $100 \%$ & $100 \%$ \\
\hline WIND (\% of assessable) & $35 \%$ & $20 \%$ & $10 \%$ & $29 \%$ & $49 \%$ & $52 \%$ & $58 \%$ & $67 \%$ & $67 \%$ & $67 \%$ & $100 \%$ \\
\hline SMOOTH (\% of assessable) & $59 \%$ & $77 \%$ & $90 \%$ & $64 \%$ & $38 \%$ & $40 \%$ & $33 \%$ & $22 \%$ & $33 \%$ & $33 \%$ & $0 \%$ \\
\hline WITH (\% of assessable) & $6 \%$ & $3 \%$ & $0 \%$ & $7 \%$ & $13 \%$ & $8 \%$ & $8 \%$ & $11 \%$ & $0 \%$ & $0 \%$ & $0 \%$ \\
\hline \multicolumn{12}{|l|}{5 DAYS } \\
\hline TOTAL EPISODES & 95 & 95 & 95 & 95 & 95 & 95 & 95 & 95 & 95 & 95 & 95 \\
\hline ASSESSABLE (\% of total) & $94 \%$ & $58 \%$ & $25 \%$ & $100 \%$ & $56 \%$ & $51 \%$ & $24 \%$ & $8 \%$ & $3 \%$ & $3 \%$ & $1 \%$ \\
\hline SUCCESS ( $\%$ of assessable) & $61 \%$ & $67 \%$ & $46 \%$ & $69 \%$ & $60 \%$ & $73 \%$ & $78 \%$ & $83 \%$ & $80 \%$ & $100 \%$ & $50 \%$ \\
\hline WIND (\% of assessable) & $35 \%$ & $22 \%$ & $9 \%$ & $20 \%$ & $50 \%$ & $53 \%$ & $61 \%$ & $70 \%$ & $75 \%$ & $50 \%$ & $100 \%$ \\
\hline SMOOTH ( $\%$ of assessable) & $56 \%$ & $76 \%$ & $91 \%$ & $77 \%$ & $36 \%$ & $40 \%$ & $32 \%$ & $20 \%$ & $25 \%$ & $50 \%$ & $0 \%$ \\
\hline WITH (\% of assessable) & $9 \%$ & $3 \%$ & $0 \%$ & $3 \%$ & $14 \%$ & $7 \%$ & $7 \%$ & $10 \%$ & $0 \%$ & $0 \%$ & $0 \%$ \\
\hline \multicolumn{12}{|l|}{10 DAYS } \\
\hline TOTAL EPISODES & 71 & 71 & 71 & 71 & 71 & 71 & 71 & 71 & 71 & 71 & 71 \\
\hline ASSESSABLE (\% of total) & $90 \%$ & $65 \%$ & $48 \%$ & $100 \%$ & $39 \%$ & $34 \%$ & $25 \%$ & $10 \%$ & $3 \%$ & $3 \%$ & $1 \%$ \\
\hline SUCCESS (\% of assessable) & $66 \%$ & $70 \%$ & $32 \%$ & $65 \%$ & $69 \%$ & $80 \%$ & $76 \%$ & $87 \%$ & $100 \%$ & $100 \%$ & $100 \%$ \\
\hline WIND (\% of assessable) & $36 \%$ & $25 \%$ & $9 \%$ & $24 \%$ & $50 \%$ & $68 \%$ & $68 \%$ & $62 \%$ & $75 \%$ & $50 \%$ & $100 \%$ \\
\hline SMOOTH (\% of assessable) & $52 \%$ & $72 \%$ & $91 \%$ & $72 \%$ & $33 \%$ & $28 \%$ & $32 \%$ & $31 \%$ & $25 \%$ & $50 \%$ & $0 \%$ \\
\hline WITH (\% of assessable $)$ & $12 \%$ & $3 \%$ & $0 \%$ & $4 \%$ & $18 \%$ & $5 \%$ & $0 \%$ & $8 \%$ & $0 \%$ & $0 \%$ & $0 \%$ \\
\hline \multicolumn{12}{|l|}{20 DAYS } \\
\hline TOTAL EPISODES & 49 & 49 & 49 & 49 & 49 & 49 & 49 & 49 & 49 & 49 & 49 \\
\hline ASSESSABLE ( $\%$ of total) & $88 \%$ & $51 \%$ & $43 \%$ & $100 \%$ & $25 \%$ & $20 \%$ & $13 \%$ & $11 \%$ & $6 \%$ & $4 \%$ & $1 \%$ \\
\hline SUCCESS (\% of assessable) & $53 \%$ & $56 \%$ & $43 \%$ & $59 \%$ & $70 \%$ & $83 \%$ & $79 \%$ & $63 \%$ & $56 \%$ & $67 \%$ & $100 \%$ \\
\hline WIND (\% of assessable) & $35 \%$ & $36 \%$ & $0 \%$ & $34 \%$ & $58 \%$ & $68 \%$ & $87 \%$ & $100 \%$ & $80 \%$ & $75 \%$ & $100 \%$ \\
\hline SMOOTH ( $\%$ of assessable) & $57 \%$ & $64 \%$ & $100 \%$ & $62 \%$ & $23 \%$ & $20 \%$ & $20 \%$ & $20 \%$ & $40 \%$ & $50 \%$ & $0 \%$ \\
\hline WITH (\% of assessable) & $9 \%$ & $0 \%$ & $0 \%$ & $3 \%$ & $19 \%$ & $12 \%$ & $0 \%$ & $0 \%$ & $0 \%$ & $0 \%$ & $0 \%$ \\
\hline \multicolumn{12}{|l|}{30 DAYS } \\
\hline TOTAL EPISODES & 46 & 46 & 46 & 46 & 46 & 46 & 46 & 46 & 46 & 46 & 46 \\
\hline ASSESSABLE ( $\%$ of total) & $87 \%$ & $48 \%$ & $39 \%$ & $100 \%$ & $22 \%$ & $18 \%$ & $11 \%$ & $9 \%$ & $7 \%$ & $5 \%$ & $3 \%$ \\
\hline SUCCESS ( $\%$ of assessable) & $58 \%$ & $64 \%$ & $50 \%$ & $41 \%$ & $82 \%$ & $74 \%$ & $81 \%$ & $62 \%$ & $64 \%$ & $71 \%$ & $40 \%$ \\
\hline WIND ( $\%$ of assessable $)$ & $35 \%$ & $36 \%$ & $0 \%$ & $47 \%$ & $56 \%$ & $75 \%$ & $85 \%$ & $100 \%$ & $86 \%$ & $80 \%$ & $50 \%$ \\
\hline SMOOTH ( $\%$ of assessable) & $57 \%$ & $64 \%$ & $100 \%$ & $47 \%$ & $19 \%$ & $20 \%$ & $15 \%$ & $13 \%$ & $29 \%$ & $40 \%$ & $50 \%$ \\
\hline WITH (\% of assessable) & $9 \%$ & 0 & $0 \%$ & $11 \%$ & $15 \%$ & $15 \%$ & $0 \%$ & $0 \%$ & $0 \%$ & $0 \%$ & $0 \%$ \\
\hline
\end{tabular}

Note: SIGNAL refers to the signaling effect, $\mathrm{W}$ in the third column refers to results obtained for the intervention episode itself (how does the exchange rate behave during the intervention episode, i.e. within the intervention window. 
Regarding unconditional exchange rate volatility measured by means of standard deviation, it is fair to say according to results reported in Table 5 that interventions are associated with both increases and decreases in volatility broadly to the same extent. The share of intervention episodes that can be associated with higher exchange rate volatility after the episode than before it, is slightly higher, and, on average is close to $60 \%$. $^{11}$

As the overall pattern emerging for episodes using different event window and pre-and postevent window sizes, is fairly comparable, in-detail results are shown only for episodes obtained on the basis of maximum 30 days of no intervention in order to save ink and paper (Tables 6 and 7). ${ }^{12}$ In yellow are marked the pre- and post event windows without any overlap with previous or forthcoming intervention episodes. A point to draw attention to is that interventions seem to be less successful in the first part of the period under study. For the second part of the period, and especially from 2002 to 2004, interventions not only appear to be more effective than previously but also the share of leaning against the wind strategy increases dramatically.

Regarding exchange rate volatility, there are episodes for which whether or not volatility increases or decreases hinges largely upon the size of the pre- and post-event window. However, in a number of episodes, interventions systematically rise volatility such (episodes No. 1, 18, 27, $28,31,32,33,43$ and 45) or dampen volatility (episodes No. 2, 8, 17, 26, 29, 37, 40 and 41)

Table 5. Intervention episodes and unconditional exchange rate volatility, Croatia EVENT SIZE

\begin{tabular}{|c|c|c|c|c|c|c|c|c|c|c|c|c|c|c|c|c|c|}
\hline & \multirow{2}{*}{\multicolumn{2}{|c|}{ WINDOW }} & & & & & & & & & & & & & \multirow{2}{*}{\multicolumn{2}{|c|}{60}} \\
\hline & & & & & 2 & & & & & & 0 & & 0 & & 0 & & \\
\hline \multirow[t]{3}{*}{2 DAYS } & & 148 & & & & & & & & & & & & & & & \\
\hline & $\mathrm{HIGH}$ & 13 & $(54 \%)$ & 74 & $(54 \%)$ & 41 & $(60 \%)$ & 18 & $(60 \%)$ & 5 & $(45 \%)$ & 1 & $(25 \%)$ & 1 & $(33 \%)$ & 0 & \\
\hline & LOW & 11 & $(46 \%)$ & 62 & $(46 \%)$ & 27 & $(40 \%)$ & 12 & $(40 \%)$ & 6 & $(55 \%)$ & 3 & $(75 \%)$ & 2 & $(67 \%)$ & 0 & \\
\hline \multirow[t]{3}{*}{5 DAYS } & & 95 & & & & & & & & & & & & & & & \\
\hline & $\mathrm{HIGH}$ & 22 & $(54 \%)$ & 44 & $(53 \%)$ & 42 & $(56 \%)$ & 22 & $(61 \%)$ & 6 & $(50 \%)$ & 2 & $(40 \%)$ & 2 & $(50 \%)$ & 2 & $(100 \%)$ \\
\hline & LOW & 19 & $(46 \%)$ & 39 & $(47 \%)$ & 33 & $(44 \%)$ & 14 & $(39 \%)$ & 6 & $(50 \%)$ & 3 & $(60 \%)$ & 2 & $(50 \%)$ & 0 & $(0 \%)$ \\
\hline \multirow[t]{3}{*}{10 DAYS } & & 71 & & & & & & & & & & & & & & & \\
\hline & HIGH & 18 & $(47 \%)$ & 32 & $(55 \%)$ & 31 & $(62 \%)$ & 24 & $(65 \%)$ & 8 & $(53 \%)$ & 2 & $(50 \%)$ & 2 & $(50 \%)$ & 1 & $(100 \%)$ \\
\hline & LOW & 20 & $(53 \%)$ & 26 & $(45 \%)$ & 19 & $(38 \%)$ & 13 & $(35 \%)$ & 7 & $(47 \%)$ & 2 & $(50 \%)$ & 2 & $(50 \%)$ & 0 & $(0 \%)$ \\
\hline \multirow[t]{3}{*}{20 DAYS } & & 49 & & & & & & & & & & & & & & & \\
\hline & $\mathrm{HIGH}$ & 23 & $(68 \%)$ & 21 & $(57 \%)$ & 18 & $(60 \%)$ & 14 & $(74 \%)$ & 11 & $(69 \%)$ & 5 & $(56 \%)$ & 4 & $(67 \%)$ & 1 & $(100 \%)$ \\
\hline & LOW & 11 & $(32 \%)$ & 16 & $(43 \%)$ & 12 & $(40 \%)$ & 5 & $(26 \%)$ & 5 & $(31 \%)$ & 4 & $(44 \%)$ & 2 & $(33 \%)$ & 0 & $(0 \%)$ \\
\hline \multirow[t]{3}{*}{30 DAYS } & & 46 & & & & & & & & & & & & & & & \\
\hline & $\mathrm{HIGH}$ & 22 & $(67 \%)$ & 18 & $(55 \%)$ & 15 & $(56 \%)$ & 10 & $(63 \%)$ & 9 & $(69 \%)$ & 7 & $(64 \%)$ & 5 & $(71 \%)$ & 4 & $(80 \%)$ \\
\hline & LOW & 11 & $(33 \%)$ & 15 & $(45 \%)$ & 12 & $(44 \%)$ & 6 & $(38 \%)$ & 4 & $(31 \%)$ & 4 & $(36 \%)$ & 2 & $(29 \%)$ & 1 & $(20 \%)$ \\
\hline
\end{tabular}

Note: as for Table 4. HIGH (LOW) indicate that exchange rate volatility higher (lower) in the post-event window as compared to the pre-event window.

\footnotetext{
${ }^{11}$ It should be noted that for single-day episodes, volatility cannot be computed and this implies that exchange rate volatility cannot be studied for the episode window.

${ }^{12}$ Results for the episodes determined on the basis of maximum 2, 5, 10 and 20 days of no interventions are available from the author upon request.
} 
Table 6. The effectiveness of intervention episodes based on maximum 30 days of no intervention, Croatia

\begin{tabular}{|c|c|c|c|c|c|c|c|c|c|c|c|c|c|c|c|c|}
\hline No. & Start & End & $\begin{array}{c}\text { Initial } \\
\text { intervention }\end{array}$ & $\begin{array}{c}\text { Total } \\
\text { intervention }\end{array}$ & $\begin{array}{c}\text { Days of } \\
\text { Interventions }\end{array}$ & $\begin{array}{l}\text { Total } \\
\text { days }\end{array}$ & $\begin{array}{l}\text { Next episode } \\
\text { (days away) }\end{array}$ & $\begin{array}{c}\text { Type of } \\
\text { intervention }\end{array}$ & window & 2 & 5 & 10 & 20 & 30 & 40 & 60 \\
\hline 1 & 10/01/1996 & 11/01/1996 & 22.6 & 30.5 & 2 & 2 & 7 & SALE & & & & & & & & \\
\hline 2 & 23/01/1996 & 01/02/1996 & -2.9 & -80.9 & 3 & 8 & 10 & PURCHASE & & & WITH & WITH & & & & \\
\hline 3 & $16 / 02 / 1996$ & $16 / 02 / 1996$ & 60.8 & 60.9 & 1 & 1 & 7 & SALE & WIND & WIND & & & & & & \\
\hline 4 & $28 / 02 / 1996$ & $06 / 03 / 1996$ & -1.1 & -16.0 & 5 & 6 & 1 & PURCHASE & & WIND & WIND & WITH & & & WITH & \\
\hline 5 & 08/03/1996 & $11 / 03 / 1996$ & 46.3 & 58.5 & 2 & 2 & 2 & SALE & WIND & & & & & & & \\
\hline 6 & $14 / 03 / 1996$ & $14 / 03 / 1996$ & -0.7 & -0.7 & 1 & 1 & 1 & PURCHASE & SMOOTH & WIND & & & & & WITH & \\
\hline 7 & 18/03/1996 & 19/03/1996 & 3.6 & 36.5 & 2 & 2 & 2 & SALE & WIND & WIND & SMOOTH & SMOOTH & SMOOTH & & & \\
\hline 8 & $22 / 03 / 1996$ & $18 / 04 / 1996$ & -4.7 & -9.1 & 4 & 19 & 5 & PURCHASE & WIND & WIND & WITH & WITH & WITH & WITH & WITH & \\
\hline 9 & $26 / 04 / 1996$ & $05 / 07 / 1996$ & 98.5 & 1058.9 & 9 & 49 & 0 & SALE & & (1) & & SMOOTH & & WT1 & WH11 & SMOOTH \\
\hline 10 & 08/07/1996 & 05/07/1996 & -0.7 & 80.3 & 2 & 2 & 4 & PURCHASE & & & & & & & & \\
\hline 11 & $12 / 07 / 1996$ & $25 / 07 / 1996$ & 234.1 & 526.0 & 2 & 10 & 0 & SALE & & SMOOTH & SMOOTH & SMOOTH & SMOOTH & WIND & SMOOTH & WIND \\
\hline 12 & $26 / 07 / 1996$ & $30 / 07 / 1996$ & -2.1 & -8.8 & 3 & 3 & 2 & PURCHASE & WITH & WITH & & WITH & & & & \\
\hline 13 & $02 / 08 / 1996$ & 21/08/1996 & 201.5 & 797.9 & 3 & 12 & 85 & SALE & WIND & & SMOOTH & WIND & WIND & WIND & WIND & WIND \\
\hline 14 & $20 / 12 / 1996$ & $20 / 12 / 1996$ & 155.7 & 155.7 & 1 & 1 & 25 & SALE & SMOOTH & SMOOTH & WIND & WIND & WIND & WIND & WIND & WIND \\
\hline 15 & $31 / 01 / 1997$ & $28 / 02 / 1997$ & -17.7 & -170.1 & 3 & 21 & 18 & PURCHASE & & WITH & & SMOOTH & SMOOTH & & & WIND \\
\hline 16 & $27 / 03 / 1997$ & $27 / 03 / 1997$ & 30.1 & 30.1 & 1 & 1 & 32 & SALE & & WIND & WIND & WIND & WITH & & & \\
\hline 17 & $15 / 05 / 1997$ & $01 / 08 / 1997$ & 70.0 & 1496.7 & 7 & 55 & 95 & SALE & & & & & & & & \\
\hline 18 & 18/12/1997 & 18/12/1997 & 223.8 & 223.8 & 1 & 1 & 94 & SALE & & SMOOTH & & & & & SMOOTH & WIND \\
\hline 19 & $08 / 05 / 1998$ & $08 / 07 / 1998$ & -38.0 & $\begin{array}{l}-682.9 \\
-10\end{array}$ & 8 & 43 & 4 & PURCHASE & SMOOTH & WIND & WIND & WIND & WIND & WIND & WIND & SMOOTH \\
\hline 20 & $15 / 07 / 1998$ & $26 / 08 / 1998$ & 48.5 & 1088.8 & 15 & 30 & 8 & SALE & & WIND & WIND & WIND & WIND & WITH & WITH & WITH \\
\hline 21 & $08 / 09 / 1998$ & $18 / 12 / 1998$ & -314.4 & -2723.2 & 16 & 74 & 1 & PURCHASE & & SMOOTH & SMOOTH & SMOOTH & SMOOTH & & & \\
\hline 22 & $22 / 12 / 1998$ & $31 / 12 / 1998$ & 1.5 & 185.4 & 4 & 7 & 7 & SALE & & WITH & WIND & WIND & WITH & WITH & WITH & WITH \\
\hline 23 & 14/01/1999 & $31 / 05 / 1999$ & -162.0 & -3481.8 & 12 & 97 & 1 & PURCHASE & & WIND & SMOOTH & SMOOTH & SMOOTH & SMOOTH & SMOOTH & WIND \\
\hline 24 & $02 / 06 / 1999$ & $02 / 06 / 1999$ & 4.4 & 4.4 & 1 & 1 & 3 & SALE & WIND & & WIND & WITH & WIND & WIND & SMOOTH & SMOOTH \\
\hline 25 & $08 / 06 / 1999$ & $08 / 06 / 1999$ & -480.6 & -480.7 & 1 & 1 & 21 & PURCHASE & WIND & SMOOTH & & & & & & \\
\hline 26 & 08/07/1999 & $02 / 09 / 1999$ & 27.4 & 182.2 & 7 & 40 & 7 & SALE & WIND & WIND & WIND & WIND & WIND & WITH & WIND & WIND \\
\hline 27 & $14 / 09 / 1999$ & $27 / 01 / 2000$ & -390.9 & -3541.8 & 11 & 93 & 55 & PURCHASE & & SMOOTH & & SMOOTH & SMOOTH & SMOOTH & SMOOTH & SMOOTH \\
\hline 28 & $17 / 04 / 2000$ & $07 / 07 / 2000$ & 180.0 & 2448.8 & 5 & 56 & 88 & SALE & & WIND & & & & & & \\
\hline 29 & $13 / 11 / 2000$ & $13 / 11 / 2000$ & -472.1 & -472.1 & 1 & 1 & 51 & PURCHASE & WIND & SMOOTH & SMOOTH & SMOOTH & & SMOOTH & & \\
\hline 30 & $29 / 01 / 2001$ & $5 / 03 / 20$ & & & 2 & 27 & 37 & PURCHASE & & WIND & SMOOTH & WIND & WIND & WIND & WINI & WIND \\
\hline 31 & $30 / 04 / 2001$ & $23 / 05 / 2001$ & 383.0 & 1142.9 & 3 & 17 & 34 & SALE & & & SMOOTH & SMOOTH & WIND & SMOOTH & SMOOTH & WIND \\
\hline 32 & $13 / 07 / 2001$ & $13 / 07 / 2001$ & 1029.0 & 1029.0 & 1 & 1 & 20 & SALE & SMOOTH & & SMOOTH & SMOOTH & WIND & WIND & WIND & WIND \\
\hline 33 & $13 / 08 / 2001$ & $31 / 08 / 2001$ & -451.1 & -3091.2 & 3 & 14 & 5 & PURCHASE & & WIND & WIND & WIND & WIND & WIND & WIND & WITH \\
\hline 34 & $10 / 09 / 2001$ & $21 / 11 / 2001$ & 970.2 & 2645.0 & 7 & 52 & 8 & SALE & & SMOOTH & WIND & WITH & & & & \\
\hline 35 & $04 / 12 / 2001$ & $04 / 12 / 2001$ & -54.4 & -54.4 & 1 & 1 & 2 & PURCHASE & SMOOTH & WIND & WIND & WIND & WITH & & & \\
\hline 36 & $07 / 12 / 2001$ & $19 / 12 / 200$ & 575.7 & 1556.1 & 3 & 9 & 7 & SALE & & SMOOTH & SMOOTH & & WIND & WIND & WIND & SMOOTH \\
\hline 37 & $03 / 01 / 2002$ & $03 / 01 / 20$ & & 373 & 1 & 1 & 9 & SALE & WITH & WITH & WITH & WIND & WIND & WIND & WIND & $\begin{array}{l}\text { WIND } \\
\text { W }\end{array}$ \\
\hline 38 & $17 / 01 / 2002$ & $31 / 01 / 2002$ & -325.5 & -1060.4 & 3 & 11 & 11 & PURCHASE & & SMOOTH & WIND & WIND & WIND & WIND & WIND & WIND \\
\hline 39 & $18 / 02 / 2002$ & $28 / 06 / 2002$ & 499.9 & 4774.6 & 10 & 91 & 32 & SALE & & WIND & SMOOTH & WIND & WIND & & & \\
\hline 40 & $16 / 08 / 2002$ & $16 / 08 / 2002$ & 347.0 & 347.0 & 1 & 1 & 47 & SALE & & SMOOTH & WIND & WIND & & & WITH & WIND \\
\hline 41 & $24 / 10 / 2$ & & -46 & -59 & 2 & 4 & 46 & ASE & SMOOTH & & & WIND & WIND & WIND & WIND & \\
\hline 42 & $09 / 01 / 20$ & $28 / 03 / 2$ & -561 & -2401 & 6 & 57 & 163 & PURCHASE & 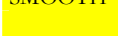 & WIND & WIND & WIND & WIND & WIND & WIND & WIND \\
\hline 43 & $21 / 11 / 2003$ & $11 / 12 / 2003$ & -458.4 & -944.3 & 2 & 15 & 8 & PURCHASE & & WIND & WIND & WIND & SMOOTH & WIND & WIND & WIND \\
\hline 44 & $24 / 12 / 2003$ & $24 / 12 / 2003$ & 646.4 & 646.4 & 1 & 1 & 51 & SALE & SMOOTH & WITH & WIND & WIND & WIND & & & \\
\hline 45 & $11 / 03 / 2004$ & $30 / 03 / 2004$ & 415.1 & 1215.1 & 4 & 14 & 64 & SALE & & WIND & WIND & WIND & WIND & & & SМООTH \\
\hline 46 & $05 / 07 / 2004$ & $17 / 09 / 2004$ & 199.3 & 1037.3 & 5 & 54 & 41 & SALE & WIND & WIND & WIND & WIND & WIND & WIND & WIND & \\
\hline
\end{tabular}


Table 7. Unconditional exchange rate volatility for intervention episodes based on maximum 30 days of no intervention, Croatia

\begin{tabular}{|c|c|c|c|c|c|c|c|c|c|c|c|c|c|c|c|c|}
\hline & Stant & Enic & $\begin{array}{l}\text { Inital } \\
\text { intervention }\end{array}$ & $\begin{array}{c}\text { 10tal } \\
\text { intervention }\end{array}$ & $\begin{array}{l}\text { Days of } \\
\text { interventions }\end{array}$ & $\begin{array}{l}\text { Iotal } \\
\text { days }\end{array}$ & $\begin{array}{l}\text { Next episode } \\
\text { (days away) }\end{array}$ & $\begin{array}{l}\text { 1ype of } \\
\text { intervention }\end{array}$ & whtow & - & & 10 & 20 & & 40 & \\
\hline 1 & 10/01/1996 & $11 / 01 / 1996$ & 22.6 & 30.5 & 2 & 2 & 7 & SALE & HIGH & $\mathrm{HIGH}$ & $\mathrm{HIGH}$ & & & & & \\
\hline 2 & $23 / 01 / 1996$ & $01 / 02 / 1996$ & -2.9 & -80.9 & 3 & 8 & 10 & PURCHASE & LOW & LOW & LOW & LOW & & & & \\
\hline 3 & $16 / 02 / 1996$ & $16 / 02 / 1996$ & 60.8 & 60.9 & 1 & 1 & 7 & SALE & & HIGH & LOW & LOW & LOW & HIGH & & \\
\hline 4 & $28 / 02 / 1996$ & $06 / 03 / 1996$ & -1.1 & -16.0 & 5 & 6 & 1 & PURCHASE & $\mathrm{HIGH}$ & LOW & HIGH & $\mathrm{HIGH}$ & $\mathrm{HIGH}$ & HIGH & HIGH & \\
\hline 5 & 08/03/1996 & $11 / 03 / 1996$ & 46.3 & 58.5 & 2 & 2 & 2 & SALE & $\mathrm{HIGH}$ & $\mathrm{HIGH}$ & HIGH & HIGH & $\mathrm{HIGH}$ & HIGH & HIGH & \\
\hline 6 & $14 / 03 / 1996$ & $14 / 03 / 1996$ & -0.7 & -0.7 & 1 & 1 & 1 & PURCHASE & & LOW & HIGH & $\mathrm{HIGH}$ & HIGH & $\mathrm{HIGH}$ & HIGH & \\
\hline 7 & $18 / 03 / 1996$ & $19 / 03 / 1996$ & 3.6 & 36.5 & 2 & 2 & 2 & SALE & HIGH & HIGH & HIGH & HIGH & HIGH & $\mathrm{HIGH}$ & HIGH & \\
\hline 8 & $22 / 03 / 1996$ & $18 / 04 / 1996$ & -4.7 & -9.1 & 4 & 19 & 5 & PURCHASE & LOW & LOW & LOW & LOW & LOW & LOW & LOW & \\
\hline 9 & $26 / 04 / 1996$ & $05 / 07 / 1996$ & 98.5 & 1058.9 & 9 & 49 & 0 & SALE & HIGH & HIGH & $\mathrm{HIGH}$ & HIGH & $\mathrm{HIGH}$ & LOW & LOW & LOW \\
\hline 10 & 08/07/1996 & 05/07/1996 & -0.7 & 80.3 & 2 & 2 & 4 & PURCHASE & HIGH & HIGH & LOW & HIGH & $\mathrm{HIGH}$ & $\mathrm{HIGH}$ & HIGH & LOW \\
\hline 11 & $12 / 07 / 1996$ & $25 / 07 / 1996$ & 234.1 & 526.0 & 2 & 10 & 0 & SALE & HIGH & LOW & LOW & LOW & LOW & LOW & LOW & LOW \\
\hline 12 & $26 / 07 / 1996$ & $30 / 07 / 1996$ & -2.1 & -8.8 & 3 & 3 & 2 & PURCHASE & HIGH & HIGH & HIGH & LOW & LOW & LOW & LOW & LOW \\
\hline 13 & $02 / 08 / 1996$ & $21 / 08 / 1996$ & 201.5 & 797.9 & 3 & 12 & 85 & SALE & LOW & HIGH & $\mathrm{HIGH}$ & $\mathrm{HIGH}$ & LOW & LOW & LOW & LOW \\
\hline 14 & $20 / 12 / 1996$ & $20 / 12 / 1996$ & 155.7 & 155.7 & 1 & 1 & 25 & SALE & & HIGH & LOW & HIGH & LOW & LOW & LOW & LOW \\
\hline 15 & $31 / 01 / 1997$ & $28 / 02 / 1997$ & -17.7 & -170.1 & 3 & 21 & 18 & PURCHASE & HIGH & HIGH & LOW & LOW & HIGH & $\mathrm{HIGH}$ & HIGH & $\mathrm{HIGH}$ \\
\hline 16 & $27 / 03 / 1997$ & $27 / 03 / 1997$ & 30.1 & 30.1 & 1 & 1 & 32 & SALE & & LOW & HIGH & $\mathrm{HIGH}$ & $\mathrm{HIGH}$ & HIGH & HIGH & HIGH \\
\hline 17 & $15 / 05 / 1997$ & $01 / 08 / 1997$ & 70.0 & 1496.7 & 7 & 55 & 95 & SALE & LOW & LOW & LOW & LOW & $\mathrm{HIGH}$ & LOW & HIGH & HIGH \\
\hline 18 & $18 / 12 / 1997$ & $18 / 12 / 1997$ & 223.8 & 223.8 & 1 & 1 & 94 & SALE & & LOW & HIGH & HIGH & HIGH & HIGH & HIGH & HIGH \\
\hline 19 & 08/05/1998 & $08 / 07 / 1998$ & -38.0 & -682.9 & 8 & 43 & 4 & PURCHASE & HIGH & LOW & LOW & HIGH & HIGH & HIGH & HIGH & HIGH \\
\hline 20 & $15 / 07 / 1998$ & $26 / 08 / 1998$ & 48.5 & 1088.8 & 15 & 30 & 8 & SALE & HIGH & HIGH & $\mathrm{HIGH}$ & HIGH & LOW & LOW & LOW & HIGH \\
\hline 21 & $08 / 09 / 1998$ & $18 / 12 / 1998$ & -314.4 & -2723.2 & 16 & 74 & 1 & PURCHASE & LOW & HIGH & LOW & LOW & LOW & LOW & LOW & LOW \\
\hline 22 & $22 / 12 / 1998$ & $31 / 12 / 1998$ & 1.5 & 185.4 & 4 & 7 & 7 & SALE & HIGH & HIGH & $\mathrm{HIGH}$ & $\mathrm{HIGH}$ & $\mathrm{HIGH}$ & HIGH & HIGH & LOW \\
\hline 23 & 14/01/1999 & $31 / 05 / 1999$ & -162.0 & -3481.8 & 12 & 97 & 1 & PURCHASE & HIGH & LOW & LOW & LOW & LOW & HIGH & HIGH & LOW \\
\hline 24 & $02 / 06 / 1999$ & $02 / 06 / 1999$ & 4.4 & 4.4 & 1 & 1 & 3 & SALE & & LOW & LOW & LOW & $\mathrm{HIGH}$ & HIGH & HIGH & HIGH \\
\hline 25 & 08/06/1999 & 08/06/1999 & -480.6 & -480.7 & 1 & 1 & 21 & PURCHASE & & LOW & LOW & $\mathrm{HIGH}$ & $\mathrm{HIGH}$ & HIGH & $\mathrm{HIGH}$ & $\mathrm{HIGH}$ \\
\hline 26 & 08/07/1999 & $02 / 09 / 1999$ & 27.4 & & 7 & 40 & 7 & SALE & LOW & LOW & LOW & LOW & LOW & HIGH & HIGH & $\mathrm{HIGH}$ \\
\hline 27 & $14 / 09 / 1999$ & $27 / 01 / 2000$ & -390.9 & -3541.8 & 11 & 93 & 55 & PURCHASE & HIGH & $\mathrm{HIGH}$ & HIGH & HIGH & $\mathrm{HIGH}$ & HIGH & HIGH & HIGH \\
\hline 28 & $17 / 04 / 2000$ & $07 / 07 / 2000$ & 180.0 & 2448.8 & 5 & 56 & 88 & SALE & HIGH & HIGH & HIGH & HIGH & $\mathrm{HIGH}$ & HIGH & HIGH & LOW \\
\hline 29 & $13 / 11 / 2000$ & $13 / 11 / 2000$ & -472.1 & -472.1 & 1 & 1 & 51 & PURCHASE & & LOW & LOW & LOW & LOW & LOW & LOW & HIGH \\
\hline 30 & $29 / 01 / 2001$ & $06 / 03 / 2001$ & -343.2 & -490.5 & 2 & 27 & 37 & PURCHASE & LOW & $\mathrm{HIGH}$ & HIGH & LOW & HIGH & HIGH & HIGH & HIGH \\
\hline 31 & $30 / 04 / 2001$ & $23 / 05 / 2001$ & 383.0 & 1142.9 & 3 & 17 & 34 & SALE & LOW & HIGH & HIGH & HIGH & HIGH & HIGH & HIGH & HIGH \\
\hline 32 & $13 / 07 / 2001$ & $13 / 07 / 2001$ & 1029.0 & 1029.0 & 1 & 1 & 20 & SALE & & HIGH & HIGH & HIGH & HIGH & HIGH & HIGH & $\mathrm{HIGH}$ \\
\hline 33 & $13 / 08 / 2001$ & $31 / 08 / 2001$ & -451.1 & $\begin{array}{r}-3091.2 \\
\end{array}$ & 3 & 14 & 5 & PURCHASE & HIGH & HIGH & $\mathrm{HIGH}$ & HIGH & LOW & LOW & LOW & LOW \\
\hline 34 & $10 / 09 / 2001$ & $21 / 11 / 2001$ & 970.2 & 2645.0 & 7 & 52 & 8 & SALE & HIGH & HIGH & LOW & LOW & LOW & LOW & LOW & LOW \\
\hline 35 & $04 / 12 / 2001$ & $04 / 12 / 2001$ & -54.4 & -54.4 & 1 & 1 & 2 & PURCHASE & & HIGH & HIGH & HIGH & $\mathrm{HIGH}$ & HIGH & HIGH & HIGH \\
\hline 36 & $07 / 12 / 2001$ & $19 / 12 / 2001$ & 575.7 & 1556.1 & 3 & 9 & 7 & SALE & LOW & LOW & LOW & LOW & HIGH & LOW & HIGH & $\mathrm{HIGH}$ \\
\hline 37 & $03 / 01 / 2002$ & $03 / 01 / 2002$ & 373.6 & 373.6 & 1 & 1 & 9 & SALE & & LOW & LOW & LOW & $\mathrm{HIGH}$ & HIGH & HIGH & HIGH \\
\hline 38 & $17 / 01 / 2002$ & $31 / 01 / 20$ & -325.5 & -106 & 3 & 11 & 11 & PURCHASE & $\mathrm{HIGH}$ & LOW & HIGH & HIGH & $\mathrm{HIGH}$ & LOW & HIGH & LOW \\
\hline 39 & $18 / 02 / 2002$ & $28 / 06 / 2002$ & 499.9 & 4774.6 & 10 & 91 & 32 & SALE & $\mathrm{HIGH}$ & HIGH & HIGH & HIGH & $\mathrm{HIGH}$ & HIGH & HIGH & $\mathrm{HIGH}$ \\
\hline 40 & $16 / 08 / 2002$ & $16 / 08 / 2002$ & 347.0 & 347.0 & 1 & 1 & 47 & SALE & & $\mathrm{HIGH}$ & LOW & LOW & LOW & LOW & LOW & LOW \\
\hline 41 & $24 / 10 / 2002$ & $29 / 10 / 2002$ & -460.6 & -597.7 & 2 & 4 & 46 & PURCHASE & $\mathrm{HIGH}$ & LOW & HIGH & LOW & LOW & LOW & LOW & LOW \\
\hline 42 & $09 / 01 / 2003$ & & -561.0 & -2401.4 & 6 & 57 & 163 & & HIGH & LO & HIGH & HIC & HIC & HIC & HIC & HIGH \\
\hline 43 & $21 / 11 / 2003$ & $11 / 12 / 2003$ & -458.4 & -944.3 & 2 & 15 & 8 & PURCHASE & HIGH & HIGH & LOW & HIGH & HIGH & HIGH & HIGH & LOW \\
\hline 44 & $24 / 12 / 2003$ & $24 / 12 / 2003$ & 646.4 & 646.4 & 1 & 1 & 51 & SALE & & LOW & LOW & LOW & LOW & LOW & LOW & $\mathrm{HIGH}$ \\
\hline 45 & $11 / 03 / 2004$ & $30 / 03 / 2004$ & 415.1 & 1215.1 & 4 & 14 & 64 & SALE & LOW & HIGH & $\mathrm{HIGH}$ & $\mathrm{HIGH}$ & $\mathrm{HIGH}$ & HIGH & HIGH & $\mathrm{HIGH}$ \\
\hline 46 & $05 / 07 / 2004$ & $17 / 09 / 2004$ & 1993 & 10373 & 5 & 54 & 41 & SALE & LOW & LOW & $\mathrm{HIGH}$ & $\mathrm{HIGH}$ & $\mathrm{HIGH}$ & $\mathrm{HIGH}$ & $\mathrm{HIGH}$ & HIGH \\
\hline
\end{tabular}




\section{Turkey}

As shown in Table 3, the number of intervention episodes in Turkey varies between 10 (maximum 2 days of no intervention) and 4 (maximum 10, 20 and 30 days of no intervention). The number of intervention episodes appears very robust to the use of the 10-day, 20-day and 30-day filters as four episodes are established in all three cases. The four episodes correspond to the four blocks of intervention activities depicted in Figure 2. During the period from 2001 to 2004, one intervention episode took place each year. In the first intervention episode in 2001, the central bank bought the Turkish lira, while in the three remaining intervention events, it sold the domestic currency against the dollar. Table 9 hereafter reports the results. In yellow are marked the pre- and post event windows without any overlap with previous or forthcoming intervention episodes. Given that 59 days elapsed without interventions after the third intervention episode, the pre- and post window size of 60 days cannot be assessed for the third and last episodes.

All four intervention episodes turn out to be very effective, and this for most pre- and post-event windows. The intervention episodes can be viewed mainly as leaning against the wind operations. Looking at the intervention windows ${ }^{13}$ indicates that the first and third intervention episodes started as exchange rate smoothing, which subsequently managed to reverse the trend of the exchange rate against the US dollar. The third episode did so only at the 2, 5 and 10-day horizons beyond which it turned out to be exchange rate smoothing.

Finally, Table 10 reports the change in the standard deviation-based exchange rate volatility between the pre- and post-event window. The first intervention episode is the only episode during and after which exchange rate volatility decreased systematically as compared to the period preceding it. During the second and last episodes, exchange rate volatility was higher both in the event window and after the intervention episode. For the third episode, volatility first declined when interventions were taking place, but then increase up to 20 days following it. For the post-event windows of 30, 40 and 60 days, it is found to be lower than in the corresponding pre-event windows.

\footnotetext{
13 This is a worthwhile undertaking because of the large size of the event windows, namely 173, 62, 121 and 63 days, respectively.
} 


\begin{tabular}{|c|c|c|c|c|c|c|c|c|}
\hline \multicolumn{9}{|c|}{ Table 8. Intervention episodes, Turkey } \\
\hline No. & Type & Beginning & End & $\begin{array}{c}\text { Initial } \\
\text { Intervention }\end{array}$ & $\begin{array}{c}\text { Total } \\
\text { intervention }\end{array}$ & $\begin{array}{c}\text { Days of } \\
\text { Interventions }\end{array}$ & $\begin{array}{c}\text { Total days of the } \\
\text { episode }\end{array}$ & $\begin{array}{l}\text { Next episode } \\
\text { (days away) }\end{array}$ \\
\hline 1 & BUY & $29 / 03 / 2001$ & $30 / 11 / 2001$ & -200 & -6553 & 124 & 173 & 77 \\
\hline 2 & SELL & $01 / 04 / 2002$ & $26 / 06 / 2002$ & 20 & 795 & 33 & 62 & 209 \\
\hline 3 & SELL & $06 / 05 / 2003$ & $22 / 10 / 2003$ & 20 & 4989 & 117 & 121 & 59 \\
\hline 4 & SELL & $23 / 01 / 2004$ & $26 / 04 / 2004$ & 30 & 2480 & 62 & 63 & 143 \\
\hline
\end{tabular}

Table 9. The effectiveness of foreign exchange interventions, Turkey

\begin{tabular}{|c|c|c|c|c|c|c|c|c|}
\hline No. Type & WINDOW & 2 & 5 & 10 & 20 & 30 & 40 & 60 \\
\hline 1 PURCHASES & SMOOTH & WIND & WIND & WIND & WITH & WIND & WIND & \\
\hline 2 SALES & WIND & & WITH & WIND & WIND & WIND & & WIND \\
\hline 3 SALES & SMOOTH & WIND & WIND & WIND & SMOOTH & SMOOTH & SMOOTH & \\
\hline 4 SALES & WIND & WIND & WIND & WIND & WIND & WIND & WIND & WIND \\
\hline
\end{tabular}

Table 10. Unconditional exchange rate volatility and the intervention episodes, Turkey

$\begin{array}{lccccccc}\text { WINDOW } & \mathbf{2} & \mathbf{5} & \mathbf{1 0} & \mathbf{2 0} & \mathbf{3 0} & \mathbf{4 0} & \mathbf{6 0} \\ 1 \text { LOW } & \text { LOW } & \text { LOW } & \text { LOW } & \text { LOW } & \text { LOW } & \text { LOW } & \text { LOW } \\ 2 \text { HIGH } & \text { HIGH } & \text { HIGH } & \text { HIGH } & \text { HIGH } & \text { HIGH } & \text { HIGH } & \text { HIGH } \\ 3 \text { LOW } & \text { HIGH } & \text { HIGH } & \text { HIGH } & \text { HIGH } & \text { LOW } & \text { LOW } & \text { LOW } \\ \text { 4 HIGH } & \text { HIGH } & \text { HIGH } & \text { HIGH } & \text { HIGH } & \text { HIGH } & \text { HIGH } & \text { HIGH } \\ \text { Note: as for Table } 4 . & & & & & & & \end{array}$

\section{Econometric Investigation 4.1. Econometric Issues}

\subsubsection{Interventions, Exchange Rates and Volatility}

In this section, the effectiveness of foreign exchange interventions is analyzed using a GARCH framework, which is admittedly well suited for such an investigation because they analyze simultaneously the mean and the conditional variance of the exchange rate series. In our economic specification, the starting point is the approach proposed by Dominguez (1998) for a $\operatorname{GARCH}(1,1)$ framework. That is, in the mean equation, the log-difference of the exchange rate ( $\Delta e_{t}$, exchange rate returns) are regressed on the intervention series $\left(I_{t}\right)$, the interest differential $\left(\Delta i_{t}\right)$ between overnight money market rates in the home economy and the foreign benchmark (Germany and the euro area for Croatia, and the US for Turkey), and dummy variables capturing day of the week effects. The conditional variance equation includes the absolute value of interventions $\left(\left|I_{t}\right|\right)$, the interest differential $\left(i_{t}-i_{t}^{*}\right)$ and day-of-the-week dummies.

Given the simultaneity problem arising between interventions and the exchange rate, lagged interventions are used instead of contemporaneous intervention. Contrary to Domac and 
Mendoza (2002) and Giumaraes and Karacadag (2004), a range of lagged interventions are employed rather than intervention with a specific lag (e.g. t-2). This does not only allow to correct for simultaneity but also helps us to identify the horizon at which interventions have an impact on the exchange rate. ${ }^{14}$ Finally, lagged values of the exchange rate are also added to the mean equation. Our baseline specification is given by equations (1a) to (3a):

$$
\begin{aligned}
& \Delta e_{t}=\phi_{1}+\sum_{i=1}^{n} \phi_{2, i} I_{t-i}+\phi_{3}\left(i_{t}-i_{t}^{*}\right)+\sum_{i=1}^{4} \phi_{4, i} D_{i}+\sum_{i=1}^{m} \phi_{5, i} \Delta e_{t-i}+\varepsilon_{t} \\
& \varepsilon_{t} \mid \Omega_{t-1} \sim N\left(0, \sigma^{2}\right) \\
& \sigma_{t}^{2}=\psi_{1}+\sum_{i=1}^{n} \psi_{2, i}\left|I_{t-i}\right|+\psi_{3}\left(i_{t}-i_{t}^{*}\right)+\sum_{i=1}^{4} \psi_{4, i} D_{i}+\alpha \varepsilon_{t-1}^{2}+\beta \sigma_{t-1}^{2}
\end{aligned}
$$

where $I_{t-i}$ takes negative (positive) values for purchases (sales) of the domestic currency. $D_{1}, D_{2}, D_{3}$ and $D_{4}$ are dummy variables that take the value of 1 on Monday, Tuesday, Wednesday and Thursday, respectively. $\varepsilon_{t-1}^{2}$ and $\sigma_{t-1}^{2}$ are the ARCH and GARCH terms. We extend this specification by distinguishing between sales and purchases as they might affect the exchange rate asymmetrically ${ }^{15}$ and, in a further step, between large and small sales and purchases ${ }^{16}$. In addition to this, two dummy variables capturing separately the length of sales $(\mathrm{SL})$ and purchases (PL) are also added $\left(D^{S L}, D^{P L}\right)$. The first dummy takes the value of 1 if a given intervention act is preceded by intervention activity in $(\mathrm{t}-1)$ and $(\mathrm{t}-2) .{ }^{17}$ In addition to this, we also use a more loosely defined dummy, which is 1 if any given intervention is preceded by intervention during one of the preceding five days, and is 0 otherwise. Equations (1b) to (3b) show the extended specification:

\footnotetext{
${ }^{14}$ An alternative approach would be to use instrumental variables for interventions. However, such an approach looks only at the contemporaneous effect and not at effects, which manifest at longer horizons. The lag length for interventions and for exchange rate returns is determined on the basis of the general-to-specific approach. We first include interventions (exchange rate returns) lagged up to ten (five) days, and decrease the maximum lag length until the last lag is found statistically significant at the $10 \%$ level.

${ }^{15}$ Domac and Mendoza (2004) and Giumaraes and Karacadag (2004) show that estimation results for the mean equation are sensitive to whether a single intervention variable containing both sales and purchases as in (1) is used or whether sales and purchases are considered separately. At the same time, the use of absolute values of interventions in the conditional variance equation studies whether higher intervention volumes lead to higher or lower exchange rate volatility. However, it may be also legitimate to think that sales and purchases may impact on exchange rate volatility in a different way.

16 Ísberg and Pétursson (2003) propose to introduce, in addition to aggregate interventions, a dummy, which takes the value of 1 if aggregate interventions are large and is 0 if they are small. However, using interventions and a closely related dummy may vehicle a very similar set of information. This is the reason we break up the intervention series directly in one series containing large interventions and another one comprising small interventions.

${ }^{17}$ Isberg and Pétursson (2003) suggested the use of this dummy variable, which captures long intervention episodes.
} 


$$
\begin{aligned}
& \Delta e_{t}=\phi_{1}+\sum_{i=1}^{n} \phi_{2,1, i} I_{t-i}^{P L}+\sum_{i=1}^{n} \phi_{2,2, i} I_{t-i}^{P S}+\sum_{i=1}^{n} \phi_{2,3, i} I_{t-i}^{S L}+\sum_{i=1}^{n} \phi_{2,4, i} I_{t-i}^{S S}+\phi_{3}\left(i_{t}-i_{t}^{*}\right)+\sum_{i=1}^{4} \phi_{4, i} D_{i}+\sum_{i=1}^{m} \phi_{5, i} \Delta e_{t-i}+\psi_{6} D^{P L}+\psi_{6} D^{S L}+\varepsilon_{t} \\
& \varepsilon_{t} \mid \Omega_{t-1} \sim N\left(0, \sigma^{2}\right) \\
& \sigma_{t}^{2}=\psi_{1}+\sum_{i=1}^{n} \psi_{2,1, i} I_{t-i}^{P L}+\sum_{i=1}^{n} \psi_{2,2, i} I_{t-i}^{P S}+\sum_{i=1}^{n} \psi_{2,3, i} I_{t-i}^{S L}+\sum_{i=1}^{n} \psi_{2,4, i} I_{t-i}^{S S}+\psi_{3}\left(i_{t}-i_{t}^{*}\right)+\sum_{i=1}^{4} \psi_{4, i} D_{i}+\psi_{6} D^{P L}+\psi_{6} D^{S L}+\alpha \varepsilon_{t-1}^{2}+\beta \sigma_{t-1}^{2}
\end{aligned}
$$

where $I^{P L}, I^{P S}, I^{S L}$ and $I^{S S}$ are large purchases (PL), small purchases (PS), large sales (SL) and small sales (SS).

The equations presented thus far rest on a $\operatorname{GARCH}(1,1)$ model. In order to check for robustness to model specification and to look at possible asymmetries in the conditional variance equation, a number of alternative GARCH models are also used for the econometric investigation. These are (a) the GARCH in mean (GARCH-M), (b) the exponential GARCH (EGARCH), (c) the threshold GARCH (TGARCH) and (d) the component GARCH (CGARCH).

For the GARCH in Mean, the only difference compared to the standard GARCH model is the inclusion of the conditional variance in the mean equation $\left(\xi \sigma_{t}^{2}\right)$. The economic interpretation of this, shown on the example of equation (1), is that exchange rate returns may depend on exchange rate volatility:

$$
\Delta e_{t}=\phi_{1}+\sum_{i=1}^{n} \phi_{2, i} I_{t-i}+\phi_{3}\left(i_{t}-i_{t}^{*}\right)+\sum_{i=1}^{4} \phi_{4, i} D_{i}+\sum_{i=1}^{m} \phi_{5, i} \Delta e_{t-i}+\xi \sigma_{t}^{2}+\varepsilon_{t}
$$

The TGARCH and EGARCH models modify the conditional variance equation in a way to account for asymmetries in the conditional variance. In addition to the standard ARCH and GARCH terms, the TGARCH model also includes a dummy term, $S_{t-1}$, that takes the value of 1 , if $\varepsilon_{t-1}<0$ (negative shock) and is 0 if $\varepsilon_{t-1}>0$ (positive shock).

$$
\sigma_{t}^{2}=\psi_{1}+\sum_{i=1}^{n} \psi_{2, i}\left|I_{t-i}\right|+\psi_{3}\left(i_{t}-i_{t}^{*}\right)+\sum_{i=1}^{4} \psi_{4, i} D_{i}+\alpha \cdot \varepsilon_{t-1}^{2}+\beta \cdot \sigma_{t-1}^{2}+\lambda \cdot \varepsilon_{t-1}^{2} \cdot S_{t-1}
$$

The impact of negative shocks on the conditional variance is higher than that of positive shocks if $\alpha+\lambda>\beta$ and is lower if $\alpha+\lambda<\beta$ provided $\lambda \neq 0$. The EGARCH is based on the log- 
transformed conditional variance, which causes the asymmetric effect to be exponential instead of being quadratic as in the TGARCH model:

$$
\log \left(\sigma_{t}^{2}\right)=\psi_{1}+\sum_{i=1}^{n} \psi_{2, i}\left|I_{t-i}\right|+\psi_{3}\left(i_{t}-i_{t}^{*}\right)+\sum_{i=1}^{4} \psi_{4, i} D_{i}+\alpha \cdot\left|\frac{\varepsilon_{t-1}}{\sigma_{t-1}}\right|+\beta \cdot \log \left(\sigma_{t-1}^{2}\right)+\lambda \cdot \frac{\varepsilon_{t-1}}{\sigma_{t-1}}
$$

The presence of asymmetry is ensured by $\lambda \neq 0$. The CGARCH model distinguishes between short- and long-term conditional volatility. Contrary to constant conditional volatility in a standard GARCH model, long-term volatility $\left(q_{t}\right)$ is allowed to vary over time, to which the short-term volatility or the transitory component of the long-term volatility $\left.\left(\sigma_{t}^{2}-q_{t}\right)\right)$ meanreverts. Such a model makes it possible to model separately the effect of interventions on exchange rate volatility in the short-run and in the long run. The short-term conditional variance model can be written as:

$$
\sigma_{t}^{2}-q_{t}=q_{t}+\psi_{1}+\sum_{i=1}^{n} \psi_{2, i}\left|I_{t-i}\right|+\psi_{3}\left(i_{t}-i_{t}^{*}\right)+\sum_{i=1}^{4} \psi_{4, i} D_{i}+\alpha \cdot\left(\varepsilon_{t-1}^{2}-q_{t}\right)+\beta \cdot\left(\sigma_{t-1}^{2}-q_{t}\right)
$$

The time-varying long-term volatility converges to $\chi$ with $\rho$ as shown in (8):

$$
q_{t}=\chi+\sum_{i=1}^{n} \psi_{2, i}\left|I_{t-i}\right|+\psi_{3}\left(i_{t}-i_{t}^{*}\right)+\sum_{i=1}^{4} \psi_{4, i} D_{i}+\rho \cdot\left(q_{t-1}-\chi\right)+\delta \cdot\left(\varepsilon_{t-1}^{2}-\sigma_{t-1}^{2}\right)
$$

\subsection{Estimation Results}

\subsubsection{Croatia}

The estimations are carried out for the whole sample from January 1996 to September 2004 and for two subperiods, namely from January 1996 to January 2000 and from April 2000 to September 2004. ${ }^{18}$ There are a number of reasons for splitting the sample: (1) the event study approach showed that interventions were more effective during the 2000s than during the late1990; (2) the visual inspection of the exchange rate and intervention series also suggests that the series are more volatile in the second half of the sample; and (3) a new governor was appointed in 2000 .

\footnotetext{
${ }^{18}$ Alternatively, the subperiods from January 1996 to February 2001 and from March 2001 to February 2004 are also analyzed. The results are very similar to those obtained for the subperiods reported in the main text.
} 
The summary of the estimation results of the different GARCH models displayed in Tables 11a to $11 \mathrm{c}$ show that the simple GARCH model appears to be sufficient to describe the data as the garch-in-mean terms $(\xi)$ - with one exception - , the asymmetric terms $(\lambda)$ of the exponential and threshold GARCH models and all structural parameters of the component GARCH model are found to be statistically insignificant. The $\mathrm{ARCH}$ and $\mathrm{GARCH}$ terms ( $\alpha$ and $\beta$ ) are strongly significant and they sum up to considerably less than one for the subperiods, indicating that the data are not fractionally integrated. Therefore, we focus on the results obtained for the simple GARCH model ${ }^{19}$.

As far as results for aggregate interventions reported in Table 11a are concerned, interventions appear to have a negative effect on exchange rate returns for the first subsample (and for the entire sample). Recalling that kuna purchases (sales) are denoted by negative (positive) values and that the exchange rate is defined as domestic currency units in terms of one unit of foreign currency, these results imply that kuna purchases (sales) associated with a depreciation (appreciation) and that interventions are either ineffective or smoothing the exchange rate. At the same time, results for the conditional variance equation show that interventions dampen exchange rate volatility. Looking at more disaggregated intervention data largely confirm these findings: interventions have mostly a negative relationship to the exchange rate. However, some qualification is needed. Large kuna purchases ${ }^{20}$ are negatively correlated with exchange rate returns, while small kuna purchases are found usually to be insignificant. Both large and small kuna sales tend to be negatively related to the exchange rate with a lag length of up to four days. However, large kuna sales with a lag of six days and small kuna sales lagged with seven days are positively associated with the exchange rate. Concerning exchange rate volatility, both large kuna purchases and large kuna sales tend to increase exchange rate volatility ${ }^{21}$. Small domestic currency sales and purchases do not have any effect on exchange rate volatility.

\footnotetext{
${ }^{19}$ Both for Croatia and Turkey, the estimations are performed for three different lag structure: for one lag for both the ARCH and the GARCH term, and for lag structures obtained using the Akaike and Schwarz information criteria by testing for a lag length of up to 6 . Because the results turn out to be fairly similar, only results based on the $(1,1)$ lag structure are reported hereafter. For a description of the data, see appendix.

${ }^{20}$ Large interventions are defined as interventions higher than the average of the interventions over the whole period, and small interventions are those below the average. For purchases (sales), average purchases (sales) are used even for aggregate intervention data. Thus, what is large is defined as compared to the average of the interventions in the same direction.

${ }^{21}$ A positive relationship between kuna purchases and forex volatility implies that kuna purchases go hand in hand with lower forex volatility. For kuna sales, a negative relationship indicates that sales go in tandem with a decrease in forex volatility.
} 
Let us now turn to the second subperiod running from 2000 to 2004. Aggregate interventions turn out to be negatively correlated with the exchange rate at short lag length ( 1 and 2), but then start having a positive impact on the exchange rate at higher lag length (6,7 and 8). No statistical relationship could be detected between aggregate interventions and forex volatility. The analysis of interventions disaggregated in large and small sales and purchases broadly confirm that interventions first have a negative effect and then kuna purchases (sales) cause the exchange rate to appreciate (depreciate). Using interventions with a lag of one day shows that both small and large purchases and sales have a negative relationship with the exchange rate, which breaks down at a lag of two days for small purchases and sales. This relationship, however, reverses quicker than for aggregate interventions, as large kuna purchases have a positive relation with the exchange rate when lagged with two and three days. This relationship disappears at a longer horizon. At the same time, small kuna sales lagged with four and six days are found to have a positive relationship with the exchange rate. Large kuna sales and small kuna purchases turn out to cause an exchange rate depreciation and appreciation at higher lag length. Combining these results with those of the event study approach would suggest that foreign exchange interventions of the Croatian National Bank first smoothes the exchange rate and at longer horizon it manages to reverse the trend.

Unlike for aggregate interventions, there appears to be some statistically significant relationship between kuna sales and purchases on the one hand, and forex volatility, on the other. Small sales, large purchases, and in particular small purchases appear to decrease forex volatility. The dummy variable aimed at capturing the duration of the intervention is either not significant or has a wrong sign.

\subsubsection{Turkey}

For Turkey, the estimations were conducted for two periods: (a) for the entire period running from 2001 to 2004 and (b) for the period from 2002 to 2004. The reason for investigating this subperiod is that, as already noted earlier, there are two clearly distinguishable subperiods. In 2001, the Central Bank of the Republic of Turkey undertook exclusively lira purchases on the foreign exchange market. During the period from 2002 to 2004, it only sold the Turkish currency against the US dollar. We decided not to use the period 2001 separately because of the relative small number of observations available for this period. 
Estimation results for the whole period are presented in Table 12a to 12c. They support the view that a simple GARCH model performs best. Only for disaggregated interventions for the whole period was found the threshold term of the exponential GARCH model to be significant, while the other models could not detect any asymmetry and garch-in-mean effect in the data.

Aggregate interventions are found to impact positively on the exchange rate with a lag of five days for the whole period. For the subperiod 2002 to 2004, they first have a negative impact on the exchange rate and then are correlated positively with the exchange rate at lag length of 2,5 and 10 days. Interventions dampen forex volatility only during the subperiod. When interventions are separated into large and small lira sales and purchases for the whole sample, the EGARCH model with the significant $\lambda$ term shows that interventions of all kind first have a negative influence on exchange rate returns, and subsequently causes the exchange rate to appreciate (lira purchases) or to depreciate (lira sales) just as expected (see third column of Table $12 \mathrm{~b})$. Nonetheless, the coefficient of both small and large lira purchases, once again, switches sign to negative at a lag length of four and seven days. As far as the relationship between interventions and forex volatility is concerned, interventions, lira purchases first dampen volatility but then are associated with an increase in forex volatility. By contrast, lira sales lead to an increase and then to a decrease in volatility as the lags increase. Taking the simple GARCH as the best model for the subperiod from 2002 to 2004 indicates that lira sales first causes the exchange rate to appreciate, then to depreciate and once again to appreciate. There seems to be no relationship between interventions and forex volatility.

Our results for Turkey are roughly in line with findings reported in Domac and (2004) who studied the period from 2001 to 2002 using EGARCH, and found that interventions had the expected effect on the exchange rate and that they lowered exchange rate volatility. However, our results are in contrast with results by Guimaraes and Karacadag (2004), who applied the asymmetric component GARCH model to data from 2001 to 2003, and found that interventions had no impact on the exchange rate and that they increased exchange rate volatility. 
Table 11a. Aggregate interventions, Croatia

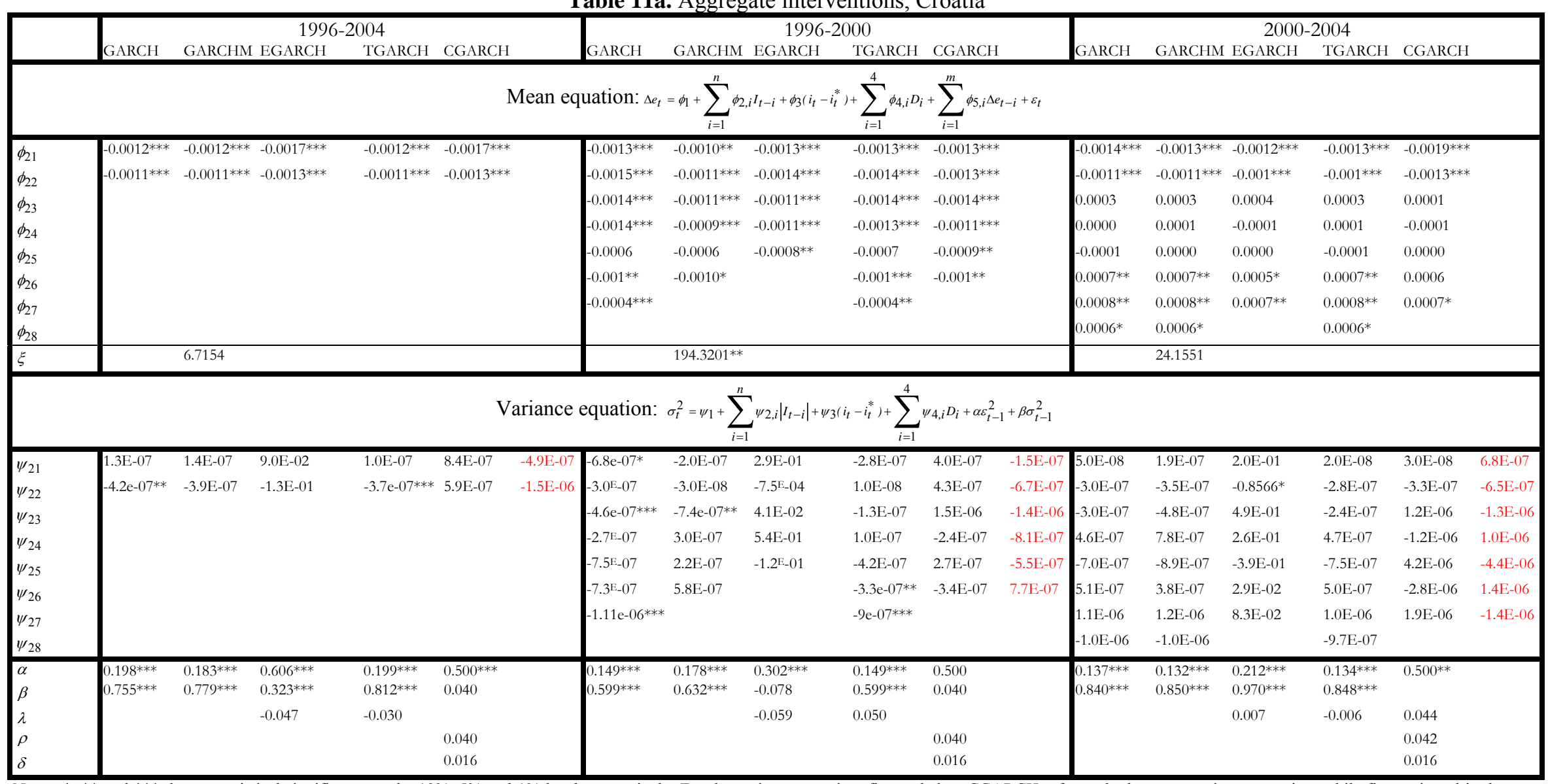

Notes: *** and $* *$ denote statistical significance at the $10 \%, 5 \%$ and $1 \%$ level, respectively. For the variance equation, figures below CGARCH refer to the long-run variance equation, while figures in red in the next

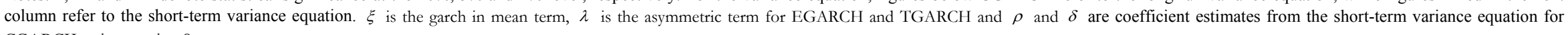
CGARCH as in equation 8 . 
Table 11b. Disaggregated interventions: mean equations, Croatia

\begin{tabular}{|c|c|c|c|c|c|c|c|c|c|c|c|c|c|c|c|}
\hline & & & 1996-2004 & & & & & $1996-2000$ & & & & & $2000-200$ & & \\
\hline & GARCH & GARCH-M & EGARCH & TGARCH & CGARCH & GARCH & GARCH-M & EGARCH & TGARCH & $\mathrm{CGARCH}$ & GARCH & GARCH-M & EGARCH & TGARCH & CGARCH \\
\hline$\phi_{211}$ & $-0.0024 * * *$ & $-0.0013^{* * *}$ & $-0.001 * * *$ & $-0.0024^{* * *}$ & $-0.0019^{* * *}$ & $-0.0011^{* * *}$ & -0.0007 & $-0.0008^{* *}$ & $-0.0011^{* * *}$ & -0.0009 & $-0.003^{* * *}$ & $-0.0019^{* * *}$ & $-0.0031 * * *$ & $-0.003 * *$ & $-0.0025^{* * *}$ \\
\hline$\phi_{221}$ & -0.0021 & -0.0013 & -0.0008 & -0.0021 & -0.0016 & -0.0011 & -0.0007 & -0.0005 & -0.0011 & -0.0009 & $-0.0083 * * *$ & $-0.005 * *$ & $-0.0077 * * *$ & $-0.0083^{* * *}$ & -0.0067 \\
\hline$\phi_{231}$ & $-0.0015^{* * *}$ & $-0.001 *$ & $-0.001 * * *$ & $-0.0015 * * *$ & $-0.0012^{* *}$ & $-0.0021 * * *$ & -0.001 & $-0.0019 * * *$ & $-0.0021 * * *$ & -0.0014 & $-0.001 *$ & -0.0008 & $-0.0012^{* * *}$ & $-0.001 *$ & -0.0009 \\
\hline$\phi_{241}$ & $-0.0025^{* *}$ & -0.0016 & -0.0004 & $-0.0025 * *$ & -0.002 & -0.0022 & -0.0011 & $-0.0023^{*}$ & -0.0022 & -0.0016 & $-0.003^{*}$ & -0.002 & $-0.0039 * * *$ & $-0.003^{*}$ & -0.0027 \\
\hline$\phi_{212}$ & $-0.0019 * * *$ & $-0.0013^{* * *}$ & $-0.0013^{* * *}$ & $-0.0019^{* * *}$ & $-0.0016^{* *}$ & $-0.0009 * * *$ & $\begin{array}{ll}-0.0006 \\
\end{array}$ & $-0.0006^{* *}$ & $-0.0009 * * *$ & $-0.0007 *$ & $-0.0025^{* * *}$ & $\begin{array}{c}-0.0015^{* *} \\
\end{array}$ & $-0.0028^{* * *}$ & $-0.0025 * * *$ & $\begin{array}{c}-0.0018^{* *} \\
\end{array}$ \\
\hline$\phi_{222}$ & $-0.0024^{*}$ & -0.0016 & -0.0013 & $-0.0024^{*}$ & -0.0019 & $-0.0023 * *$ & -0.0014 & $-0.0018^{* *}$ & $-0.0023^{* *}$ & $-0.0019 *$ & -0.0052 & -0.0026 & -0.0034 & -0.0052 & -0.0035 \\
\hline$\phi_{232}$ & $-0.0009 * * *$ & $-0.0008^{* * *}$ & $-0.001 * * *$ & $-0.0009 * * *$ & $-0.0007 *$ & -0.0006 & -0.0003 & $-0.0016^{* * *}$ & -0.0006 & -0.0004 & $-0.0008^{* *}$ & -0.0006 & $-0.0007 * *$ & $-0.0008 * *$ & -0.0007 \\
\hline$\phi_{242}$ & $-0.0039 * *$ & $-0.0025 * *$ & $-0.0044 * * *$ & $-0.0039 * * *$ & $-0.0031 *$ & $-0.0054 * * *$ & -0.0029 & $-0.007 * * *$ & $-0.0054 * * *$ & $-0.004 *$ & -0.0023 & -0.0013 & -0.0009 & -0.0023 & -0.0017 \\
\hline$\phi_{213}$ & 0.0002 & 0.0000 & $\begin{array}{l}-0.0002 \\
\end{array}$ & 0.0002 & 0.0001 & $-0.0009 * *$ & -0.0005 & $-0.001 * * *$ & $-0.0008^{* *}$ & -0.0007 & $0.0011^{* * *}$ & $0.0006 * *$ & $0.0012^{* *}$ & $0.0011^{* * *}$ & 0.0007 \\
\hline$\phi_{223}$ & -0.0022 & -0.0015 & -0.001 & -0.0022 & -0.0018 & $-0.0035 * * *$ & -0.0021 & $-0.0038 * * *$ & $-0.0035 * * *$ & $-0.0027 *$ & 0.0037 & 0.0022 & 0.0036 & 0.0037 & 0.003 \\
\hline$\phi_{233}$ & -0.0007 & -0.0006 & -0.0003 & -0.0007 & -0.0005 & $-0.0009 *$ & -0.0006 & -0.0007 & $-0.001 * *$ & -0.0008 & -0.0006 & -0.0002 & -0.0003 & -0.0006 & -0.0003 \\
\hline$\phi_{243}$ & 0.0002 & 0.0001 & -0.0009 & 0.0002 & 0.0002 & $-0.0033 * * *$ & -0.0019 & $-0.0033^{* * *}$ & $-0.0033^{* * *}$ & $-0.0026^{*}$ & 0.0038 & 0.0023 & $0.006 * * *$ & 0.0038 & 0.0031 \\
\hline$\phi_{214}$ & 0.0009 & 0.0004 & 0 & 0.0009 & 0.0007 & -0.0005 & -0.0004 & -0.0005 & -0.0005 & $\begin{array}{l}-0.0004 \\
\end{array}$ & $0.0017 * * *$ & $0.001 * *$ & $0.0013^{* * *}$ & $0.0017 * * *$ & 0.0013 \\
\hline$\phi_{224}$ & -0.0001 & -0.0001 & -0.001 & -0.0001 & -0.0001 & -0.0016 & -0.001 & -0.0009 & -0.0016 & -0.0012 & 0.0016 & 0.0009 & -0.0025 & 0.0016 & 0.0012 \\
\hline$\phi_{234}$ & $-0.0009 *$ & $-0.0007 *$ & -0.0006 & $-0.0009 *$ & -0.0008 & $-0.0024 * * *$ & -0.0016 & $-0.0018 * * *$ & $-0.0024 * * *$ & -0.002 & -0.0006 & -0.0004 & -0.0003 & -0.0006 & -0.0005 \\
\hline$\phi_{244}$ & -0.0008 & -0.0006 & $-0.0026^{* *}$ & -0.0008 & -0.0006 & $-0.0048 * * *$ & -0.0028 & $-0.0036^{* *}$ & $-0.0048 * * *$ & $-0.0038 * *$ & $0.0031 *$ & 0.0019 & 0.0011 & 0.0031 & 0.0025 \\
\hline$\phi_{215}$ & 0.0004 & 0.0001 & -0.0003 & 0.0004 & 0.0003 & -0.0008 & -0.0005 & -0.0005 & -0.0008 & -0.0007 & $\begin{array}{l}0.0009 \\
\end{array}$ & 0.0005 & 0.0006 & 0.0008 & 0.0007 \\
\hline$\phi_{225}$ & 0.0007 & 0.0003 & -0.0008 & 0.0007 & 0.0005 & -0.001 & -0.0006 & -0.0009 & -0.001 & -0.0008 & 0.0061 & 0.0039 & 0.0064 & 0.0061 & 0.0052 \\
\hline$\phi_{235}$ & -0.0003 & -0.0002 & -0.0003 & -0.0003 & -0.0002 & 0.0003 & 0.0002 & -0.0007 & 0.0003 & 0.0003 & -0.0001 & -0.0002 & -0.0003 & -0.0001 & -0.0002 \\
\hline$\phi_{245}$ & 0.002 & 0.0011 & $0.002^{* *}$ & $0.002^{*}$ & 0.0016 & -0.0004 & -0.0005 & -0.0005 & -0.0004 & -0.0004 & 0.0022 & 0.0014 & 0.0013 & 0.0022 & 0.0018 \\
\hline$\phi_{216}$ & 0.0002 & 0.0000 & $\begin{array}{l}-0.0003 \\
\end{array}$ & 0.0002 & 0.0002 & $-0.0012^{* * *}$ & $\begin{array}{l}-0.0007 \\
\end{array}$ & $-0.001^{* * *}$ & $-0.0012^{* * *}$ & $\begin{array}{l}-0.001 * \\
\end{array}$ & 0.0007 & 0.0004 & 0.0003 & 0.0007 & 0.0005 \\
\hline$\phi_{226}$ & -0.001 & -0.0007 & -0.0011 & -0.001 & -0.0008 & -0.002 & -0.0012 & $-0.0026 * *$ & -0.002 & -0.0016 & -0.0003 & -0.0002 & 0.0001 & -0.0003 & -0.0002 \\
\hline$\phi_{236}$ & 0.0006 & 0.0003 & 0.0001 & 0.0006 & 0.0004 & $0.0012^{* * *}$ & 0.0007 & $0.0019 * * *$ & $0.0012^{* * *}$ & 0.001 & 0.0006 & 0.0003 & 0.0004 & 0.0006 & 0.0004 \\
\hline$\phi_{246}$ & 0.001 & 0.0005 & -0.001 & 0.001 & 0.0008 & -0.0014 & -0.0011 & -0.0021 & -0.0014 & -0.0012 & $0.0034 * *$ & 0.0017 & $0.0032^{* *}$ & $0.0034 *$ & 0.0023 \\
\hline$\phi_{217}$ & 0.0002 & -0.0001 & -0.0002 & 0.0002 & 0.0002 & $-0.0007 * * *$ & & $-0.0007 * *$ & $-0.0007 * * *$ & & 0.0006 & 0.0004 & 0.0005 & 0.0006 & 0.0005 \\
\hline$\phi_{227}$ & 0.0017 & 0.001 & 0.0015 & 0.0017 & 0.0014 & 0.0008 & & 0.0003 & 0.0008 & & -0.0011 & -0.0006 & 0.0007 & -0.0011 & -0.0009 \\
\hline$\phi_{237}$ & $0.0011^{* * *}$ & $0.0006^{* * *}$ & $0.0007 *$ & $0.0011 * * *$ & $0.0009 * *$ & -0.0004 & & -0.0001 & -0.0004 & & $0.0012^{* * *}$ & $0.0007 *$ & $0.0011^{* * *}$ & $0.0012^{* * *}$ & $0.001 *$ \\
\hline$\phi_{247}$ & $0.0023^{*}$ & 0.0014 & 0.0018 & 0.0023 & 0.0018 & $0.0022^{*}$ & & $0.0025 * *$ & $0.0022^{*}$ & & 0.0014 & 0.0008 & 0.001 & 0.0014 & 0.001 \\
\hline$\phi_{218}$ & $0.0003^{*}$ & 0.0004 & & $0.0003^{*}$ & 0.0003 & & & $-0.0004^{*}$ & & & 0.0005 & & 0.0004 & 0.0005 & \\
\hline$\phi_{228}$ & 0.0001 & -0.0001 & & 0.0001 & 0.0001 & & & $-0.0017 * *$ & & & $0.0091 * * *$ & & $0.0097 * * *$ & $0.0091 * * *$ & \\
\hline$\phi_{238}$ & $0.001 * *$ & $0.0007 * *$ & & $0.001 * *$ & $0.0008^{*}$ & & & 0.0009 & & & $0.0011^{* * *}$ & & $0.0009 * *$ & $0.0011^{* * *}$ & \\
\hline$\phi_{248}$ & -0.0001 & -0.0001 & & -0.0001 & -0.0001 & & & $0.003^{*}$ & & & -0.0042 & & $-0.0046 * *$ & -0.0041 & \\
\hline$\phi_{6}$ & $\overline{0.0002}$ & 0.0000 & -0.0001 & 0.0002 & 0.0002 & 0.0000 & 0.0000 & 0.0001 & 0.0000 & 0.0001 & $0.0016^{* *}$ & 0.001 & $0.0017^{* * *}$ & $0.0015^{* *}$ & 0.0013 \\
\hline$\phi_{7}$ & 0.0001 & 0.0000 & 0.0000 & 0.0001 & 0.0001 & 0.0002 & 0.0001 & 0 & 0.0002 & 0.0001 & 0.0001 & 0.0001 & 0.0001 & 0.0001 & 0.0001 \\
\hline$\xi$ & & 0.5707 & & & & & 210.951 & & & & & 8.5294 & & & \\
\hline
\end{tabular}


Table 11c. Disaggregated interventions: variance equations, Croatia

\begin{tabular}{|c|c|c|c|c|c|c|c|c|c|c|c|c|c|c|c|c|c|c|}
\hline & \multicolumn{6}{|c|}{ 1996-2004 } & \multicolumn{6}{|c|}{$1996-2000$} & \multicolumn{6}{|c|}{$2000-2004$} \\
\hline & GARCH & GARCH-M & EGARCH & TGARCH & CGARCH & & GARCH & GARCH-M & EGARCH & TGARCH & CGARCH & & GARCH & GARCH-M & EGARCH & TGARCH & CGARCH & \\
\hline$\psi_{211}$ & $6.5 \mathrm{E}-07$ & $-1.8 \mathrm{E}-07$ & -0.2936 & $5.8 \mathrm{E}-07$ & $-1.2 \mathrm{E}-07$ & $-1.4 \mathrm{E}-07$ & $6 \mathrm{e}-07 * * *$ & $8.5 \mathrm{E}-07$ & -0.2669 & $6.4 \mathrm{e}-07 * * *$ & $-3.0 \mathrm{E}-08$ & $0.0 \mathrm{E}+00$ & $1.2 \mathrm{E}-06$ & $1.4 \mathrm{E}-06$ & 0.3023 & $1.2 \mathrm{E}-06$ & $-7 \mathrm{E}-08$ & 3.6E-06 \\
\hline$\psi_{221}$ & 2.4E-06 & $1 \mathrm{E}-06$ & 0.5828 & 2.3E-06 & $1.6 \mathrm{E}-06$ & $1.5 \mathrm{E}-06$ & 5E-07 & $1.4 \mathrm{E}-06$ & -0.8780 & 7.3E-07 & 2.6E- 07 & $-5.9 \mathrm{E}-07$ & $1.452 \mathrm{e}-05^{* * *}$ & $1.587 \mathrm{e}-05^{* * *}$ & $8.8537 * * *$ & $1.061 \mathrm{e}-05^{* * *}$ & $2.7 \mathrm{E}-06$ & 6E-08 \\
\hline$\psi_{231}$ & $-9.1 \mathrm{E}-07$ & $1.7 \mathrm{E}-07$ & 0.0740 & $-8.8 \mathrm{E}-07$ & $6 \mathrm{E}-08$ & $1.7 \mathrm{E}-07$ & $-1.95 \mathrm{e}-06^{* * *}$ & $-6.5 \mathrm{E}-07$ & $-2.9106^{* *}$ & $-2.18 \mathrm{e}-06 * * *$ & $9.0 \mathrm{E}-08$ & $-1.2 \mathrm{E}-06$ & $-7.8 \mathrm{E}-07$ & $-8.3 \mathrm{E}-07$ & 0.4111 & $-8.3 \mathrm{E}-07$ & $-7 \mathrm{E}-08$ & $-1.3 \mathrm{E}-06$ \\
\hline$\psi_{241}$ & $-2.6 \mathrm{E}-06$ & $-1.8 \mathrm{E}-06$ & -0.4053 & $-3.31 \mathrm{e}-06^{*}$ & $-2.2 \mathrm{E}-07$ & $-6.8 \mathrm{E}-07$ & $-4 \mathrm{E}-07$ & $-5.2 \mathrm{E}-07$ & -0.6020 & $-7.1 \mathrm{E}-07$ & $1.4 \mathrm{E}-06$ & $-1.1 \mathrm{E}-06$ & $-6.31 \mathrm{e}-06 * * *$ & $-6.5 \mathrm{E}-06$ & $-6.2316^{* * *}$ & $-6.24 \mathrm{e}-06 * * *$ & $-1.3 \mathrm{E}-06$ & $-4 \mathrm{E}-08$ \\
\hline$\psi_{212}$ & $3.9 \mathrm{E}-07$ & $6.9 \mathrm{E}-07$ & 0.6540 & $3.1 \mathrm{E}-07$ & $-2 \mathrm{E}-08$ & $-3 \mathrm{E}-08$ & $6.6 \mathrm{e}-07 * * *$ & $1.1 \mathrm{E}-06$ & $1.8153^{* *}$ & $8.3 \mathrm{e}-07 * * *$ & $3.0 \mathrm{E}-07$ & $4.5 \mathrm{E}-07$ & $6.9 \mathrm{e}-07 * *$ & $1.2 \mathrm{E}-06$ & -0.2144 & $6.8 \mathrm{E}-07$ & $-4 \mathrm{E}-08$ & $3.1 \mathrm{E}-06$ \\
\hline$\psi 222$ & 2.1E-06 & $8 \mathrm{E}-07$ & 2.1771 & $2.1 \mathrm{E}-06$ & $9.1 \mathrm{E}-07$ & $1 \mathrm{E}-06$ & $1 \mathrm{E}-06$ & $7.8 \mathrm{E}-07$ & 2.9128 & $1.1 \mathrm{E}-06$ & 4.5E- 07 & $9.9 \mathrm{E}-07$ & $2.8 \mathrm{E}-07$ & $5.82 \mathrm{e}-06 *$ & 4.7200 & $3.2 \mathrm{E}-06$ & $3.8 \mathrm{E}-06$ & $-6.6 \mathrm{E}-07$ \\
\hline$\psi_{232}$ & $-1.3 \mathrm{E}-06$ & $-1.56 \mathrm{e}-06^{*}$ & -0.7173 & $-1.4 \mathrm{E}-06$ & $-2.1 \mathrm{E}-07$ & $-4.7 \mathrm{E}-07$ & $1.2 \mathrm{E}-06$ & $3 \mathrm{E}-08$ & 0.1868 & $1.1 \mathrm{E}-06$ & $2.3 \mathrm{E}-07$ & $5.0 \mathrm{E}-07$ & $-8.8 \mathrm{E}-07$ & $-1 \mathrm{E}-06$ & $-1.7076^{* *}$ & $-9.4 \mathrm{E}-07$ & $-4.1 \mathrm{E}-07$ & $-1.1 \mathrm{E}-06$ \\
\hline$\psi_{242}$ & $-1.1 \mathrm{E}-06$ & 3E-08 & 0.7112 & $-9.9 \mathrm{E}-07$ & $2 \mathrm{E}-07$ & $-2 \mathrm{E}-07$ & $1.7 \mathrm{E}-06$ & $1.5 \mathrm{E}-07$ & 2.2332 & 2.3E-06 & $9.7 \mathrm{E}-07$ & $8.3 \mathrm{E}-07$ & $-1.7 \mathrm{E}-06$ & $-5.67 \mathrm{e}-06^{* *}$ & $-4.3919 *$ & $-2.4 \mathrm{E}-06$ & $-5.4 \mathrm{E}-07$ & $-1 \mathrm{E}-08$ \\
\hline$\psi_{213}$ & $6.8 \mathrm{E}-07$ & $8.6 \mathrm{e}-07^{* *}$ & 0.3611 & 6E-07 & 5E-08 & $9 \mathrm{E}-08$ & $6 \mathrm{E}-08$ & 4.4E-07 & -0.0424 & $-5.0 \mathrm{E}-08$ & $-1.1 \mathrm{E}-07$ & $2.8 \mathrm{E}-07$ & $7.9 \mathrm{E}-07$ & $1.68 \mathrm{e}-06^{*}$ & -0.3793 & $7.8 \mathrm{E}-07$ & $-2 \mathrm{E}-08$ & $4.1 \mathrm{E}-06$ \\
\hline$\psi_{223}$ & 2.4E-06 & $1.7 \mathrm{E}-07$ & -3.1115 & $2.4 \mathrm{E}-06$ & $7.3 \mathrm{E}-07$ & 3.3E-07 & $4.1 \mathrm{E}-07$ & $6.9 \mathrm{E}-07$ & -1.2753 & 5.6E- 07 & $-5.3 \mathrm{E}-07$ & $-6.2 \mathrm{E}-07$ & $5.21 \mathrm{e}-06^{*}$ & $4 \mathrm{E}-06$ & $7.0140^{* * *}$ & $4.5 \mathrm{E}-06$ & $2.7 \mathrm{E}-06$ & $-3 \mathrm{E}-08$ \\
\hline$\psi_{233}$ & $-3.6 \mathrm{E}-07$ & $6.2 \mathrm{E}-07$ & 0.4277 & $-3 \mathrm{E}-07$ & $5 \mathrm{E}-08$ & $3 \mathrm{E}-08$ & $-1.4 \mathrm{E}-06$ & $-7.1 \mathrm{E}-07$ & 0.0576 & $-1.6 \mathrm{E}-06$ & $-2.9 \mathrm{E}-07$ & $1.3 \mathrm{E}-07$ & $-5.3 \mathrm{E}-07$ & $-6.9 \mathrm{E}-07$ & -0.4358 & $-5.8 \mathrm{E}-07$ & $-4 \mathrm{E}-08$ & $5.7 \mathrm{E}-07$ \\
\hline$\psi_{243}$ & $-1.4 \mathrm{E}-06$ & $4 \mathrm{E}-06$ & 1.1525 & $-1.5 \mathrm{E}-06$ & 7.4E- 07 & $5.2 \mathrm{E}-07$ & $-7.4 \mathrm{E}-07$ & $-1.7 \mathrm{E}-06$ & -2.4618 & $-1.5 \mathrm{E}-06$ & 6.6E- 07 & $-1.2 \mathrm{E}-06$ & 1.7E-06 & $5.3 \mathrm{E}-07$ & 2.2791 & $1.8 \mathrm{E}-07$ & 3.4E- 07 & $2.7 \mathrm{E}-07$ \\
\hline$\psi 214$ & 4.2E- 07 & $-9 \mathrm{E}-08$ & -0.5676 & 4E-07 & $-4 \mathrm{E}-08$ & $1.4 \mathrm{E}-07$ & $1.8 \mathrm{E}-07$ & $4.9 \mathrm{E}-07$ & 0.5235 & $3.1 \mathrm{E}-07$ & $1.4 \mathrm{E}-07$ & 1.6E- 07 & $2.9 \mathrm{E}-07$ & $5.2 \mathrm{e}-07 * *$ & 0.2514 & 4.6E-07 & 0 & $-2 \mathrm{E}-07$ \\
\hline$\psi 224$ & 2.1E-06 & $9.9 \mathrm{E}-07$ & 0.7567 & $2.1 \mathrm{E}-06$ & 7.2E-07 & $5.2 \mathrm{E}-07$ & 4.9E-07 & $8.8 \mathrm{E}-07$ & -0.7646 & $6.9 \mathrm{E}-07$ & $-1.1 \mathrm{E}-07$ & $5.0 \mathrm{E}-08$ & $-2.6 \mathrm{E}-07$ & $4.5 \mathrm{E}-06$ & 0.9250 & $1.5 \mathrm{E}-06$ & $6.2 \mathrm{E}-07$ & $-1 \mathrm{E}-08$ \\
\hline$\psi_{234}$ & $-2 \mathrm{E}-07$ & 4.3E-07 & 0.1169 & $-2.5 \mathrm{E}-07$ & $1 \mathrm{E}-07$ & $1.1 \mathrm{E}-07$ & $-6.7 \mathrm{e}-07 *$ & $-6.3 \mathrm{E}-07$ & -2.1190 & $-7.2 \mathrm{e}-07 * * *$ & $-4.6 \mathrm{E}-07$ & $-8.6 \mathrm{E}-07$ & $-3.8 \mathrm{E}-07$ & $-7.9 \mathrm{E}-07$ & -0.0066 & $-5 \mathrm{E}-07$ & $-8 \mathrm{E}-08$ & $-8.8 \mathrm{E}-07$ \\
\hline$\psi_{244}$ & $-1.4 \mathrm{E}-06$ & $4.2 \mathrm{E}-06$ & 1.8354 & $-1.6 \mathrm{E}-06$ & $5.5 \mathrm{E}-07$ & $6.2 \mathrm{E}-07$ & 5.4E-06 & $1.8 \mathrm{E}-06$ & $7.0331 * * *$ & $5.0 \mathrm{E}-06$ & $6.8 \mathrm{E}-07$ & $4.1 \mathrm{E}-07$ & $-4.1 \mathrm{E}-06$ & $-2.7 \mathrm{E}-06$ & -2.7652 & $-2.3 \mathrm{E}-06$ & $-4.5 \mathrm{E}-07$ & $2 \mathrm{E}-08$ \\
\hline$\psi_{215}$ & 4.6E- 07 & $5 \mathrm{E}-08$ & -0.0586 & $6.1 \mathrm{E}-07$ & $-9 \mathrm{E}-08$ & 0 & 6E-08 & $3.8 \mathrm{E}-07$ & -0.9053 & $1.4 \mathrm{E}-07$ & $6.9 \mathrm{E}-07$ & $-1.3 \mathrm{E}-06$ & $1.8 \mathrm{E}-07$ & $-1.2 \mathrm{E}-07$ & 0.1569 & $2.3 \mathrm{E}-07$ & $-1.2 \mathrm{E}-07$ & $-3.5 \mathrm{E}-07$ \\
\hline$\psi_{225}$ & 2.1E-06 & $-4.4 \mathrm{E}-07$ & 1.7068 & $1.9 \mathrm{E}-06$ & $1.1 \mathrm{E}-06$ & 1.6E-06 & $9.1 \mathrm{E}-07$ & $1.1 \mathrm{E}-06$ & $3.5604^{* *}$ & $9.0 \mathrm{E}-07$ & $3.5 \mathrm{E}-07$ & $1.3 \mathrm{E}-06$ & 4.1E- -06 & 7.4e- $06^{*}$ & -3.3926 & 4.6E-06 & $1.3 \mathrm{E}-06$ & $-7 \mathrm{E}-08$ \\
\hline$\psi_{235}$ & $-1.3 \mathrm{E}-06$ & $-7.8 \mathrm{E}-07$ & -0.2963 & $-1.1 \mathrm{E}-06$ & $3 \mathrm{E}-08$ & 6E-08 & 4E-07 & $-8.2 \mathrm{E}-07$ & 1.5057 & 4.4E- 07 & $1.3 \mathrm{E}-06$ & $2.0 \mathrm{E}-07$ & $-7.4 \mathrm{E}-07$ & $-1.2 \mathrm{E}-06$ & -0.4905 & $-8.1 \mathrm{E}-07$ & $-1.9 \mathrm{E}-07$ & $3 \mathrm{E}-07$ \\
\hline$\psi_{245}$ & $-2.4 \mathrm{E}-06$ & $-6.8 \mathrm{E}-06$ & -3.3579 & $-2.3 \mathrm{E}-06$ & $-2.5 \mathrm{E}-07$ & $-5.9 \mathrm{E}-07$ & -5.9E-06 & $-2.6 \mathrm{E}-06$ & -2.1366 & $-4.8 \mathrm{E}-06$ & $2.8 \mathrm{E}-06$ & $-3.7 \mathrm{E}-06$ & $-1.2 \mathrm{E}-07$ & $-3 \mathrm{E}-08$ & 0.1970 & $-1.1 \mathrm{E}-07$ & 1.6E-07 & $-1.1 \mathrm{E}-07$ \\
\hline$\psi 216$ & 6.9E-07 & $-7.4 \mathrm{E}-07$ & -0.1981 & $8.5 \mathrm{E}-07$ & $-1.3 \mathrm{E}-07$ & $-1.5 \mathrm{E}-07$ & $5.4 \mathrm{E}-07$ & $7.3 \mathrm{e}-07^{*}$ & 0.7727 & $5.8 \mathrm{e}-07 * *$ & $8.7 \mathrm{E}-07$ & $-7.3 \mathrm{E}-07$ & $3 \mathrm{E}-08$ & $-7.9 \mathrm{E}-07$ & -1.3075 & $8 \mathrm{E}-08$ & $-1.1 \mathrm{E}-07$ & $3.9 \mathrm{E}-06$ \\
\hline$\psi 226$ & $1.8 \mathrm{E}-06$ & $-5.3 \mathrm{E}-07$ & -3.0279 & $1.8 \mathrm{E}-06$ & $1.5 \mathrm{E}-07$ & $3 \mathrm{E}-08$ & $-1 \mathrm{E}-06$ & 3.6E-07 & -2.4689 & $-8.1 \mathrm{E}-07$ & $-1.7 \mathrm{E}-06$ & $7.0 \mathrm{E}-07$ & $1.062 \mathrm{e}-05 * *$ & $1.287 \mathrm{e}-05^{* * *}$ & $13.6143 * * *$ & $1.029 \mathrm{e}-05 * *$ & $4 \mathrm{E}-06$ & $-6.1 \mathrm{E}-07$ \\
\hline$\psi 236$ & $-6 \mathrm{E}-07$ & $-8 \mathrm{e}-07 *$ & -0.4620 & $-6 \mathrm{E}-07$ & $-9 \mathrm{E}-08$ & $-3.8 \mathrm{E}-07$ & $-1.4 \mathrm{E}-06$ & $-1.4 \mathrm{E}-06$ & -0.6580 & $-1.5 \mathrm{E}-06$ & $9.3 \mathrm{E}-07$ & $-2.2 \mathrm{E}-06$ & $-8.3 \mathrm{E}-07$ & $-1.1 \mathrm{E}-06$ & $-1.0801 * * *$ & $-8.6 \mathrm{E}-07$ & $-4.3 \mathrm{E}-07$ & $-1.9 \mathrm{E}-06$ \\
\hline$\psi_{246}$ & $-1.7 \mathrm{E}-06$ & 3.4E-06 & 2.0021 & $-1.5 \mathrm{E}-06$ & 3.3E-07 & $1.9 \mathrm{E}-07$ & 2.3E-06 & $5.2 \mathrm{E}-07$ & 1.9545 & $1.5 \mathrm{E}-06$ & $5.2 \mathrm{E}-06$ & $-4.5 \mathrm{E}-06$ & $-7 \mathrm{e}-06^{*}$ & $-4.9 \mathrm{E}-06$ & $-4.7825^{* * *}$ & $-5.1 \mathrm{E}-06$ & $-1 \mathrm{E}-06$ & $5.6 \mathrm{E}-07$ \\
\hline$\psi 217$ & 9.3E-07 & $1.39 \mathrm{e}-06^{* *}$ & 0.2137 & $1.1 \mathrm{E}-06$ & $2.2 \mathrm{E}-07$ & $6.2 \mathrm{E}-07$ & $8.5 \mathrm{e}-07^{* * *}$ & & 1.4001 & $8.8 \mathrm{e}-07^{* * *}$ & & & $6.3 \mathrm{E}-07$ & $3.5 \mathrm{E}-07$ & $2.0646^{* * *}$ & $6.6 \mathrm{E}-07$ & $3 \mathrm{E}-08$ & 4E-06 \\
\hline$\psi_{227}$ & 2.5E-06 & $8.8 \mathrm{E}-07$ & 1.2838 & $2.4 \mathrm{E}-06$ & 7.4E-07 & 7.6E-07 & $2.8 \mathrm{E}-07$ & & -2.7660 & 4.7E- 07 & & & $5.97 \mathrm{e}-06 * *$ & $7.27 \mathrm{e}-06 * * *$ & $12.2735 * * *$ & $6.25 \mathrm{e}-06 * * *$ & $1.9 \mathrm{E}-06$ & $3 \mathrm{E}-08$ \\
\hline$\psi_{237}$ & $-4.9 \mathrm{E}-07$ & 3.9E-07 & $0.7242 *$ & $-5 \mathrm{E}-07$ & $1.9 \mathrm{E}-07$ & 2.4E- 07 & 8.3E- 07 & & -1.5961 & $6.5 \mathrm{E}-07$ & & & $-7.5 \mathrm{E}-07$ & $-8.7 \mathrm{E}-07$ & 0.2470 & $-7.4 \mathrm{E}-07$ & $-3 \mathrm{E}-08$ & $1.5 \mathrm{E}-06$ \\
\hline$\psi_{247}$ & $-1.4 \mathrm{E}-06$ & $3.8 \mathrm{E}-07$ & -0.8755 & $-1.2 \mathrm{E}-06$ & 3.6E-07 & 3.1E-07 & $-3.2 \mathrm{E}-07$ & & 0.2141 & $-4.6 \mathrm{E}-07$ & & & 3.4E-06 & $-3.9 \mathrm{E}-07$ & 1.7334 & $1.1 \mathrm{E}-06$ & $7.5 \mathrm{E}-07$ & \\
\hline$\psi 218$ & 4.2E- -07 & $-4.7 \mathrm{E}-07$ & & $4.1 \mathrm{E}-07$ & $-1 \mathrm{E}-07$ & $-1.7 \mathrm{E}-07$ & & & 1.2083 & & & & 3.7E-07 & & -0.7837 & $4.2 \mathrm{E}-07$ & & \\
\hline$\psi_{228}$ & 2.18e- $06 * *$ & $9.4 \mathrm{E}-07$ & & $2.2 \mathrm{E}-06$ & 2.4E-07 & 3.3E-07 & & & 3.0921* & & & & $4.01 \mathrm{e}-06^{*}$ & & 6.5411 & $4.15 \mathrm{e}-06^{*}$ & & \\
\hline$\psi_{238}$ & $-1.09 \mathrm{e}-06^{*}$ & $-3.3^{\mathrm{E}}-07$ & & $-9.4 \mathrm{E}-07$ & $-2 \mathrm{E}-08$ & $2 \mathrm{E}-08$ & & & 2.6312 & & & & $-6.9 \mathrm{E}-07$ & & -0.3190 & $-6.5 \mathrm{E}-07$ & & \\
\hline$\psi_{248}$ & $-1.8 \mathrm{E}-06$ & $3 \mathrm{E}-08$ & & $-1.8 \mathrm{E}-06$ & 2.4E- 07 & $1.2 \mathrm{E}-07$ & & & 3.0562 & & & & $-2.4 \mathrm{E}-06$ & & 0.5173 & $-2.8 \mathrm{E}-06$ & & $1.2 \mathrm{E}-06$ \\
\hline$\psi_{6}$ & $-2.9 \mathrm{E}-07$ & $\begin{array}{l}-4 \mathrm{E}-08 \\
\end{array}$ & 0.0272 & $-2.8 \mathrm{E}-07$ & $-8 \mathrm{E}-08$ & $-1 \mathrm{E}-07$ & $-2 \mathrm{E}-08$ & $-1 \mathrm{E}-08$ & $\begin{array}{l}-0.4241^{* *} \\
\end{array}$ & $-4.0 \mathrm{E}-08$ & $0.0 \mathrm{E}+00$ & $-2.1 \mathrm{E}-07$ & $-8.6 \mathrm{E}-07$ & $-1.59 \mathrm{e}-06^{*}$ & -0.3768 & $-1.36 \mathrm{e}-06^{*}$ & $-4.2 \mathrm{E}-07$ & $-1.3 \mathrm{E}-07$ \\
\hline$\psi_{7}$ & $-1.5 \mathrm{E}-07$ & $1.1 \mathrm{E}_{-} 07$ & 0.0166 & $-1.3 \mathrm{E}-07$ & $-1 \mathrm{E}-08$ & $3 \mathrm{E}-08$ & $2 \mathrm{E}-08$ & $-9 \mathrm{E}-08$ & -0.1273 & $-2.0 \mathrm{E}-08$ & $-3.9 \mathrm{E}-07$ & 2.4E-07 & $-4 \mathrm{E}-07$ & $-5.3 \mathrm{E}-07$ & 0.2370 & $-4.7 \mathrm{E}-07$ & $-1.5 \mathrm{E}-07$ & 0 \\
\hline$\alpha$ & $0.149^{* * *}$ & $0.150^{* * *}$ & $0.215^{* * *}$ & $0.149 * * *$ & 0.500 & & $0.149 * * *$ & $0.149 * * *$ & $0.431 * * *$ & $0.149 * * *$ & 0.500 & & $0.149 * * *$ & $0.149 * * *$ & $0.491 * * *$ & $0.149 * * *$ & 0.500 & \\
\hline$\beta$ & $0.599 * * *$ & $0.599 * * *$ & $0.981 * * *$ & $0.599 * * *$ & 0.040 & & $0.599 * * *$ & $0.599 * * *$ & $0.278^{*}$ & $0.599 * * *$ & 0.040 & & $0.599 * * *$ & $0.599 * * *$ & 0.054 & $0.599 * * *$ & 0.040 & \\
\hline$\lambda$ & & & 0.009 & 0.050 & & & & & -0.002 & 0.050 & & & & & -0.016 & 0.050 & & \\
\hline$\rho$ & & & & & 0.040 & & & & & & 0.040 & & & & & & 0.040 & \\
\hline & & & & & 0.016 & & & & & & 0.016 & & & & & & 0.016 & \\
\hline
\end{tabular}


Table 12a. Aggregate interventions, Turkey

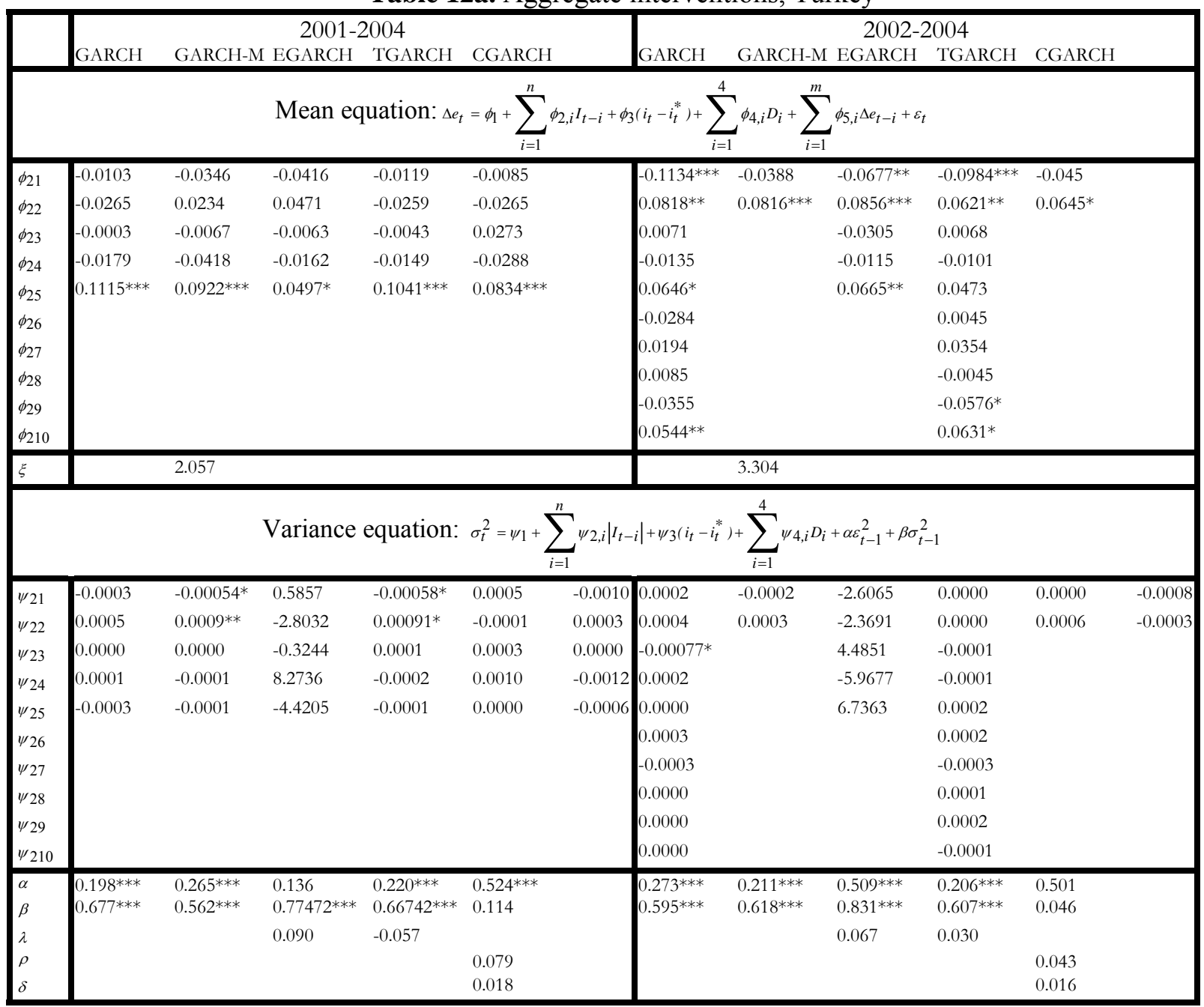

Notes: as for Table 11a. 
Table 12b. Disaggregated interventions, Turkey, 2001-2004

\begin{tabular}{|c|c|c|c|c|c|c|c|c|c|c|c|c|}
\hline & GARCH & GARCHM & EGARCH & TGARCH & CGARCH & & GARCH & GARCHM & EGARCH & TGARCH & CGARCH & \\
\hline$\phi_{211}$ & 0.0291 & 0.0021 & $-0.0544 * * *$ & 0.0287 & 0.0143 & $\psi_{211}$ & -0.00065 & -0.00007 & 3.23191 & -0.00076 & -0.00011 & -0.00015 \\
\hline$\phi_{221}$ & -0.2869 & -0.1916 & 0.0926 & -0.287 & -0.2476 & $\psi_{221}$ & -0.00031 & -0.00201 & -14.78531 & -0.00091 & -0.00013 & -0.00023 \\
\hline$\phi_{231}$ & $-0.0926 * * *$ & -0.039 & $-0.0763^{* * *}$ & $-0.0925^{* * *}$ & -0.0732 & $\psi_{231}$ & -0.00008 & -0.00014 & 2.41959 & -0.00008 & -0.00005 & -0.00006 \\
\hline$\phi_{241}$ & $-0.0977 *$ & -0.0487 & $-0.0686^{* *}$ & $-0.0976^{*}$ & -0.0696 & $\psi_{241}$ & -0.00028 & -0.00062 & -14.57193 & -0.00025 & -0.00024 & -0.00029 \\
\hline$\phi_{212}$ & -0.1189 *** & -0.0595 & -0.0081 & $-0.1191^{* * *}$ & $-0.0773^{* *}$ & $\psi_{212}$ & -0.00154 & $-0.00394 *$ & $-10.16289 * * *$ & -0.00202 & -0.00011 & -0.00013 \\
\hline$\phi_{222}$ & 0.0771 & 0.0384 & $-0.1499 *$ & 0.0771 & 0.0801 & $\psi_{222}$ & -0.00035 & -0.00059 & -13.06938 & -0.00094 & -0.00013 & -0.00025 \\
\hline$\phi_{232}$ & $0.0862 * * *$ & $0.051^{*}$ & $0.1067^{* * *}$ & $0.0858^{* * *}$ & 0.0563 & $\psi_{232}$ & -0.00011 & -0.00004 & 2.41778 & -0.0001 & -0.00005 & -0.00006 \\
\hline$\phi_{242}$ & 0.0494 & -0.0034 & 0.0173 & 0.0498 & 0.0058 & $\psi_{242}$ & -0.00022 & -0.00017 & $-17.66701 * *$ & -0.00022 & -0.0002 & -0.00028 \\
\hline$\phi_{213}$ & 0.0521 & 0.0119 & 0.0161 & 0.0518 & 0.0199 & $\psi_{213}$ & 0.00013 & -0.00013 & $-7.65482 * * *$ & -0.00015 & -0.00018 & -0.0002 \\
\hline$\phi_{223}$ & 0.2051 & 0.1279 & $0.2075^{* * *}$ & 0.2051 & 0.1742 & $\psi_{223}$ & -0.00012 & 0.00116 & -11.66949 & 0.00006 & -0.0001 & -0.00016 \\
\hline$\phi_{233}$ & 0.0091 & 0.0043 & -0.0266 & 0.0089 & 0.003 & $\psi_{233}$ & -0.00017 & -0.00011 & -8.8831 & -0.00017 & -0.00004 & -0.00004 \\
\hline$\phi_{243}$ & 0.0411 & 0.0358 & -0.0167 & 0.0412 & 0.0336 & $\psi_{243}$ & -0.00022 & 0.00077 & $32.16906^{* * *}$ & -0.00025 & -0.00012 & -0.00014 \\
\hline$\phi_{214}$ & $-0.0945^{* *}$ & -0.046 & 0.0195 & $-0.0944 * *$ & -0.0533 & $\psi_{214}$ & 0.00046 & $0.00218^{*}$ & 5.11656 & 0.00059 & -0.00016 & -0.00012 \\
\hline$\phi_{224}$ & -0.0469 & 0.0288 & $-0.104 * * *$ & -0.0468 & -0.0266 & $\psi_{224}$ & -0.00014 & -0.0014 & -3.70408 & -0.00029 & -0.0001 & -0.00018 \\
\hline$\phi_{234}$ & -0.0189 & 0.0189 & 0.0353 & -0.0187 & 0.0046 & $\psi_{234}$ & -0.00018 & 0.00012 & $-11.21732 *$ & -0.0002 & -0.00006 & -0.00011 \\
\hline$\phi_{244}$ & 0.0007 & 0.0271 & 0.0259 & 0.0009 & 0.0242 & $\psi_{244}$ & -0.00021 & -0.00041 & 2.7804 & -0.00024 & -0.00008 & 0 \\
\hline$\phi_{215}$ & $0.1715^{* * *}$ & $0.0741^{* *}$ & -0.0119 & $0.1717 * * *$ & 0.1159 ** & $\psi_{215}$ & -0.00027 & -0.00085 & 2.0997 & -0.00022 & -0.00033 & -0.00034 \\
\hline$\phi_{225}$ & -0.0109 & 0.0752 & -0.0448 & -0.0108 & -0.0061 & $\psi_{225}$ & -0.00006 & 0.00142 & -0.46829 & -0.00006 & -0.0001 & -0.00016 \\
\hline$\phi_{235}$ & 0.0576 & 0.0579 & 0.0335 & 0.0573 & 0.0382 & $\psi 235$ & -0.00017 & 0.0001 & 6.76607 & -0.00022 & -0.00004 & -0.00006 \\
\hline$\phi_{245}$ & 0.0522 & 0.0403 & $0.1578^{* *}$ & 0.0522 & 0.0206 & $\psi_{245}$ & -0.00021 & 0.00004 & $-20.08794^{*}$ & -0.00024 & -0.00015 & -0.00019 \\
\hline$\phi_{216}$ & -0.0231 & & $0.0466^{* * *}$ & -0.0229 & $\begin{array}{l}-0.0048 \\
\end{array}$ & $\psi_{216}$ & 0.00069 & -0.00002 & $15.61577^{* * *}$ & 0.0007 & -0.00023 & -0.00001 \\
\hline$\phi_{226}$ & 0.1032 & & 0.0449 & 0.1032 & -0.0264 & $\psi_{226}$ & -0.00007 & -0.00001 & 23.11917* & -0.00028 & -0.0001 & -0.00017 \\
\hline$\phi_{236}$ & $0.0696 * *$ & & $0.0464^{*}$ & $0.0697^{* *}$ & 0.0539 & $\psi_{236}$ & -0.00011 & $0 * * *$ & $20.67539 * * *$ & -0.00008 & -0.00002 & -0.00002 \\
\hline$\phi_{246}$ & -0.0047 & & -0.0579 & -0.0047 & -0.022 & $\psi_{246}$ & -0.00021 & $-9 \mathrm{e}-05^{* * *}$ & $34.73022^{* * *}$ & -0.00023 & -0.00009 & 0 \\
\hline$\phi_{217}$ & 0.0177 & & $-0.0759 * * *$ & 0.0179 & $\begin{array}{l}-0.0138 \\
\end{array}$ & $\psi_{217}$ & -0.00006 & $-9 \mathrm{e}-05^{* * *}$ & $-11.95601 * * *$ & 0.00019 & -0.00019 & -0.00029 \\
\hline$\phi_{227}$ & $0.3472^{*}$ & & 0.1318 & $0.3472^{*}$ & 0.1897 & $\psi_{227}$ & 0.00008 & $-6 \mathrm{e}-05^{* * *}$ & 7.05865 & 0.0001 & -0.00008 & -0.00011 \\
\hline$\phi_{237}$ & 0.0024 & & 0.0082 & 0.0028 & 0.0119 & $\psi_{237}$ & -0.00012 & $-4 \mathrm{e}-05^{* *}$ & 3.24445 & -0.00017 & -0.00003 & -0.00004 \\
\hline$\phi_{247}$ & $0.2131 * * *$ & & $0.1276^{* * *}$ & $0.2132 * * *$ & $0.1263^{*}$ & $\psi_{247}$ & -0.00022 & & $26.11285^{* *}$ & -0.00024 & -0.00018 & -0.00007 \\
\hline$\phi_{218}$ & $-0.0642^{*}$ & & $-0.0302^{* *}$ & $-0.0646^{*}$ & & $\psi_{218}$ & 0.0001 & & 0.84433 & 0.00009 & & \\
\hline$\phi_{228}$ & -0.1718 & & -0.1334 & -0.1717 & & $\psi_{228}$ & 0.00024 & & 17.00718 & 0.00046 & & \\
\hline$\phi_{238}$ & 0.0052 & & -0.0033 & 0.005 & & $\psi_{238}$ & -0.00008 & & -0.95435 & -0.00011 & & \\
\hline$\phi_{248}$ & $-0.149 * *$ & & $-0.0957^{* *}$ & $-0.1488 * *$ & & $\psi_{248}$ & -0.00025 & & $-29.84616 * * *$ & -0.00026 & & \\
\hline$\phi_{219}$ & $0.058^{*}$ & & & $0.0577^{*}$ & & $\psi_{219}$ & 0.00024 & & 0.00189 & 0.00039 & & \\
\hline$\phi_{229}$ & -0.1749 & & & -0.175 & & $\psi_{229}$ & 0.00011 & & -0.49003 & 0.00013 & & \\
\hline$\phi_{239}$ & $-0.0649^{*}$ & & & $-0.065^{*}$ & & $\psi 239$ & -0.00003 & & $0.03197 * * *$ & 0.00001 & & \\
\hline$\phi_{249}$ & $-0.1041 *$ & & & $-0.104 * *$ & & $\psi 249$ & -0.0003 & & $0.35727^{*}$ & -0.0003 & & \\
\hline$\phi_{2110}$ & $-0.0621 * *$ & & & $-0.0621^{* *}$ & & $\psi 2110$ & -0.00057 & & -0.19444 & -0.00077 & & \\
\hline$\phi_{2210}$ & 0.114 & & & 0.1139 & & $\psi_{2210}$ & 0.00031 & & 0.04063 & 0.00052 & & \\
\hline$\phi_{2310}$ & $0.067^{*}$ & & & $0.0668^{*}$ & & $\psi 2310$ & -0.00002 & & 0.13617 & 0.00002 & & \\
\hline$\phi_{2410}$ & $0.1394 * *$ & & & $0.1396 * * *$ & & $\psi_{2410}$ & -0.00032 & & & -0.00031 & & \\
\hline $\begin{array}{l}\phi_{6} \\
\phi_{7}\end{array}$ & $\begin{array}{l}0.0009 \\
-0.0027 *\end{array}$ & $\begin{array}{l}0.0007 \\
-0.0021\end{array}$ & $\begin{array}{l}-0.0008 \\
-0.003^{* * *}\end{array}$ & $\begin{array}{l}0.0009 \\
-0.0026^{*}\end{array}$ & $\begin{array}{l}0.0014 \\
-0.0022\end{array}$ & $\begin{array}{l}\psi_{6} \\
\psi_{7}\end{array}$ & $\begin{array}{l}-0.00004 \\
-0.00001\end{array}$ & & & $\begin{array}{l}-6 \mathrm{e}-05^{*} \\
-0.00001\end{array}$ & $\begin{array}{l}0.00001 \\
0\end{array}$ & $\begin{array}{l}0.00002 \\
0\end{array}$ \\
\hline $\begin{array}{l}\xi \\
\alpha \\
\beta \\
\lambda \\
\rho \\
\delta\end{array}$ & & 1.3821 & & & & $\begin{array}{l}\xi \\
\alpha \\
\beta \\
\lambda \\
\rho \\
\delta\end{array}$ & $\begin{array}{l}0.1474^{* * *} \\
0.586^{* * *}\end{array}$ & $\begin{array}{l}0.159^{* * *} \\
0.585^{* * *}\end{array}$ & $\begin{array}{l}0.679^{* * *} \\
0.316^{* * *} \\
0.206^{* * *}\end{array}$ & $\begin{array}{l}0.148^{* * *} \\
0.586^{* * *} \\
0.047\end{array}$ & $\begin{array}{l}0.49997 \\
0.04024 \\
0.04013 \\
0.01599\end{array}$ & \\
\hline
\end{tabular}

Notes: as for Table 11a. 
Table 12c. Disaggregated interventions, Turkey, 2002-2004

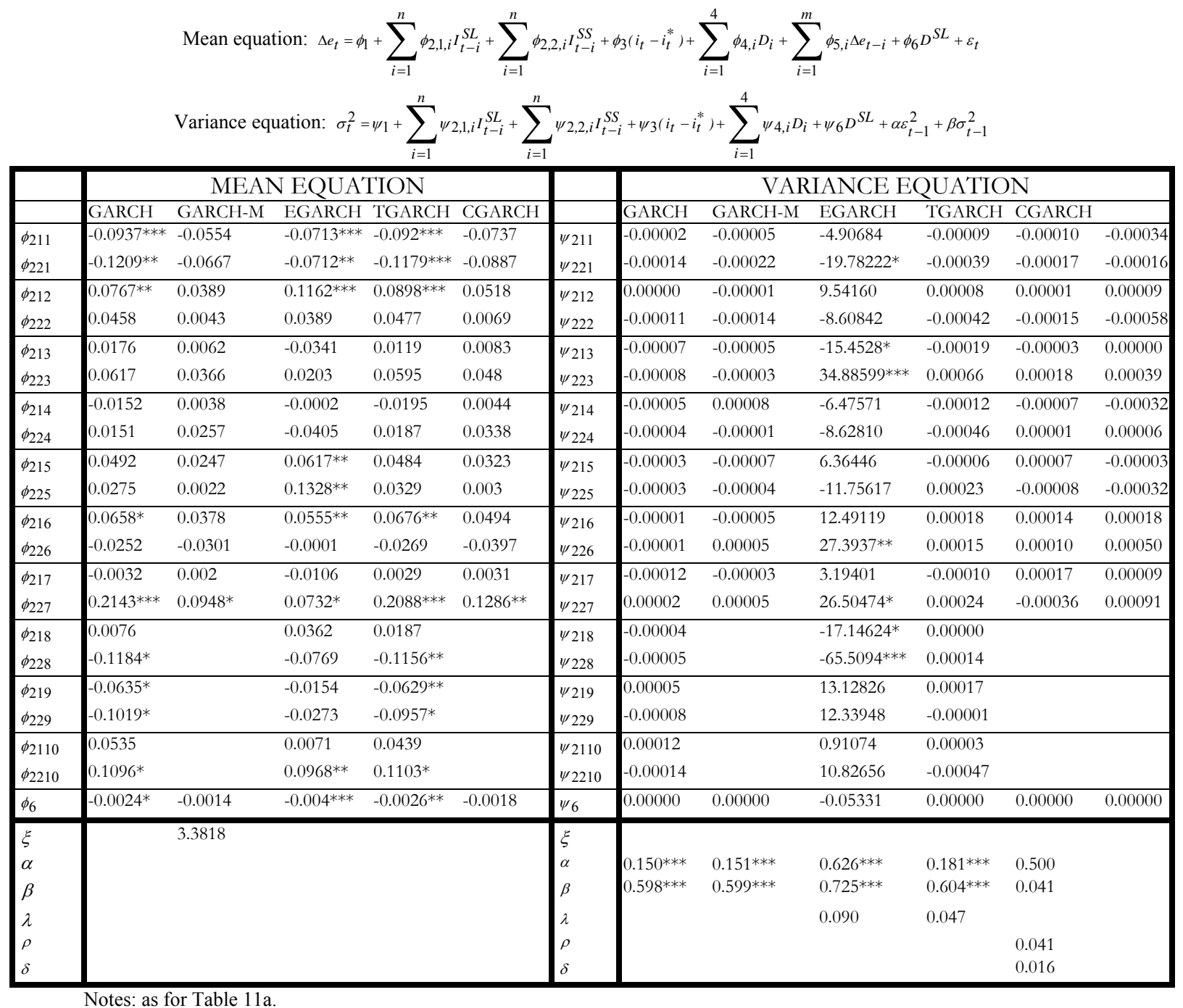

\section{Conclusions}

This paper investigated the impact of foreign exchange interventions in two EU candidate countries, namely in Croatia and Turkey by applying the event study methodology to the data, and by analyzing a variety of the class of GARCH models. The results indicate that the event study and the econometric estimations are complementary rather than competing approaches.

The event study approach can be used to analyze only a fraction of foreign exchange interventions by the Croatian National Bank because of its regular interventions leaving little time to elapse between two interventions (episodes). For the non-overlapping intervention episodes, the rate of success was found to vary between $60 \%$ and $80 \%$. The successful episodes 
were mainly leaning against the wind and exchange rate smoothing. Some qualification merits mention. First, it appeared that the share of exchange rate smoothing was higher than that of leaning against the wind in the direct aftermath of the intervention episodes. However, the share of leaning against the wind steadily increases for larger post-event windows. This implies that interventions (kuna purchases/sales) first start to decrease the pace of depreciation/appreciation, and as time goes by, they even manage to change the trend on the foreign exchange market. Second, interventions appear to be more effective from 2000/2001 to 2004 than during the late 1990s. In addition, some evidence is also found for the existence of a signaling channel.

The econometric estimations confirm the earlier finding that interventions are more effective during the second subperiod. They also reveal that the dynamics of the different types of interventions (small and large kuna sales and purchases) varies considerably. Nonetheless, the general pattern that emerges is that interventions first have a negative relationship with the exchange rate, which reverses when interventions with higher lags are used. This is broadly in line with findings of the event study approach and suggests that kuna sales/purchases dampen the pace of the kuna appreciation/depreciation in a first stage (exchange rate smoothing) and subsequently are able to reverse the trend of the exchange rate (leaning against the wind). For forex volatility, some evidence is found for that interventions are associated with lower volatility. During the late 1990s, large kuna sales and purchases are found to decrease forex volatility, while from 2000 to 2004, small kuna sales and purchases tended to be correlated with lower forex volatility.

For Turkey, the event study approach revealed that the Central Bank of the Republic of Turkey intervened periodically, but massively on the foreign exchange market. The identified four intervention episodes are all found to have been very effective. The Turkish central bank always acted as leaning against the wind, and the interventions had a significant impact on changes in the exchange rate against the dollar to a horizon up to 60 days. Regarding unconditional exchange rate volatility, the first intervention episode occurred in 2001 in the aftermath of the currency crisis clearly dampened volatility, whilst the episodes in 2002 and 2004 turn out to have led to higher volatility with comparison to the pre-event volatility. The third intervention episode taking place in 2003 first increased volatility but then resulted in lower volatility at the horizons of 30 to 60 days. 
Although the results of the GARCH estimations are less convincing than those of the event study approach, they also suggest that foreign exchange interventions were successful at specific lag length to change the trend of the exchange rate. The analysis of the conditional variance for the whole period indicated that lira purchases are associated first with a decrease and then with an increase in volatility. By contrast, lira sales appeared to generate more volatility in a first step, followed by some dampening effect on forex volatility. However, no relationship could be detected for the subperiod.

Combining the results of the event study and the econometric estimations suggest that both lira purchases and lira sales were successful, to some extent, in changing the trend of exchange rate movements (leaning against the wind) and lowering exchange rate volatility. These findings underline that the official policy statements of the Central Bank of the Republic of Turkey, according to which the aim of forex interventions is to decrease forex volatility but not to affect the level of the exchange rate, are somewhat in contrast with the achievements. The question that could be asked is whether there is perhaps too much exchange rate targeting and insufficient volatility targeting?

Overall, the results of this study gives further evidence in favor of the fact that emerging market economies are in a good position to carry out (sterilized) foreign exchange interventions effectively. 


\section{References}

Aguilar, Javiera and Stefan Nydahl (2000): Central bank intervention and exchange rates: The case of Sweden, Journal of International Financial Markets, Institutions and Money, 10, 303-322.

Brandner, P. and H. Grech (2002): Why did central banks intervene in the EMS? The post 1993 experience, Oesterreichische Nationalbank Working Paper No. 77.

Brandner, P., Grech H and H. Stix (2001): The effectiveness of central bank intervention in the EMS: The post 1993 experience, Oesterreichische Nationalbank Working Paper No. 55.

Brissimis, Sophocles N. and Dionysios P. Chionis (2004): Foreign exchange market intervention: implications of publicly announced and secret intervention for the euro exchange rate and its volatility, Journal of Policy Modeling, 26, 661-673.

Calvo, G.A. and C.M. Reinhart (2000): Fear of floating, NBER Working Paper No. 7993.

Canales-Kriljenko, Jorge Iván (2003): Foreign exchange intervention in developing and transition economies: Results of a survey, IMF Working Paper No. 95.

Canales-Kriljenko, Jorge-Iván, Roberto Guimaraes and Cem Karacadag (2003): Official intervention in the foreign exchange market: Elements of best practice, IMF Working Paper No 152.

Central Bank of Turkey (2001, 2002, 2003): Annual Report

Central Bank of Turkey (2004): General framework of monetary and exchange rate policy in 2004, Press Release No. 2.

Croatian National Bank (2001, 2002, 2003): Annual Report.

Domac, Ilker and Alfonso Mendoza (2004): Is there room for forex interventions under inflation targeting framework? Evidence from Mexico and Turkey, World Bank Policy Research Working Paper No. 3288.

Dominguez, Kathryn M. (1998): Central bank intervention and exchange rate volatility, Journal of International Money and Finance, 17, 161-190.

Dutta, Jayasri and Leon Hyginus (2002): Dread of depreciation: Measuring real exchange rate interventions, IMF Working Paper No. 63.

Edison, Hali, Paul Cashin and Hong Liang (2003): Foreign exchange intervention and the Australian dollar: Has it mattered? IMF Working Paper No 99.

Fatum, Rasmus (2000): On the effectiveness of sterilised Foreign Exchange Intervention, ECB Working Paper No. 10.

Fatum, Rasmus and Michael M. Hutchison (2003): Effectiveness of official daily foreign exchange market intervention operations in Japan, NBER Working Paper No. 9648.

Guimaraes, Roberto and Cem Karacadag (2004): The empirics of foreign exchange intervention in emerging market countries: The case of Mexico and Turkey, IMF Working Paper 123.

Kearns, Jonathan and Roberto Rigobon (2002): Identifying the efficacy of central bank interventions: The Australian case, NBER Working Paper No. 9062.

Kim, Suk-Joong, Tro Kortian and Jeffrey Sheen (2000): Central bank intervention and exchange rate volatility Australian evidence, Journal of International Financial Markets, Institutions and Money, 10, 381-405.

Mihaljek, Dubravko (2005): Survey of central banks' views on effects of intervention. Bank for International Settlements, mimeo.

Morana, Claudia and Andrea Beltratti (2000): Central bank interventions and exchange rates: an analysis with high frequency data, , Journal of International Financial Markets, Institutions and Money, 10, 349-362.

Ramaswamy, Ramana and Hossein Samiei (2000): The yen-dollar rate: Have interventions mattered? IMF Working Paper No. 95.

Rogers, J.M. and P.L. Siklos (2003): Foreign exchange market intervention in two small open economies: The Canadian and Australian experience, Journal of International Money and Finance, 22, 393-416.

Sarno, Lucio and Mark P. Taylor (2001): Official intervention in the foreign exchange market: Is it effective, and, if so, how does it work? CEPR Discussion Paper No. 2690. 


\section{Data Appendix}

Daily intervention data are obtained from the national central banks. The sample period spans from January 1996 to September 2004 for Croatia and from January 2001 to June 2004 for Turkey. The interventions are expressed in the domestic currencies (billions of Croatia kuna) for Croatia because the sample period comprises the switch from the German mark to the euro. Expressing interventions in the same currency units ensures full comparability. The intervention series are Interventions by the Central Bank of the Republic of Turkey are expressed in terms of billions of US dollar. In accordance with common practice in the literature, purchases (sales) of the foreign currency are positive (negative) values. Thus, purchases (sales) of the domestic currencies are denoted with negative (positive) figures. Exchange rate series against the German mark and the euro are used for Croatia (provided by the respective central banks, and against the US dollar for Turkey (obtained from Datastream, code: TKUSDSP). Exchange rates are defined

as units of the domestic currency per one unit of the foreign currency. This implies that a decrease (increase) in the exchange rate is an appreciation (depreciation). The exchange rate returns series are obtained as first differences of the level series taken in natural logs. Only data for trading days are considered for the study implying the exclusion of weekends and public holidays.

For the interest rates, overnight money market rates are used. The data for Croatia and Germany are obtained from the Croatian National Bank and the Bundesbank, whilst the data for Turkey and the US are drawn from Bloomberg.

Large interventions are defined as interventions higher than the average of the interventions over the whole period, and small interventions are those below the average. For purchases (sales), average purchases (sales) are used even for aggregate intervention data. Thus, what is large is defined as compared to the average of the interventions in the same direction. 


\section{DAVIDSON INSTITUTE WORKING PAPER SERIES - Most Recent Papers}

The entire Working Paper Series may be downloaded free of charge at: www.wdi.bus.umich.edu

CURRENT AS OF 4/08/05

\begin{tabular}{|c|c|c|}
\hline Publication & Authors & Date \\
\hline $\begin{array}{l}\text { No. 764: State Regulations, Job Search and Wage Bargaining: A Study } \\
\text { in the Economics of the Informal Sector }\end{array}$ & Maxim Bouev & Apr. 2005 \\
\hline $\begin{array}{l}\text { No. 763: The Feldstein-Horioka Puzzle Revisited: An “European- } \\
\text { Regional” Perspective }\end{array}$ & $\begin{array}{l}\text { Jérôme Hericourt and Mathilde } \\
\text { Maurel }\end{array}$ & Apr. 2005 \\
\hline $\begin{array}{l}\text { No. 762: Transatlantic Differences in Labour Markets Changes in Wage } \\
\text { and Non-Employment Structures in the 1980s and the 1990s }\end{array}$ & Patrick A. Puhani & Mar. 2005 \\
\hline $\begin{array}{l}\text { No. 761: Resolution, Recovery and Survival: The Evolution of Payment } \\
\text { Disputes in Post-Socialist Europe }\end{array}$ & William Pyle & Mar. 2005 \\
\hline $\begin{array}{l}\text { No. 760: Official Foreign Exchange Interventions in the Czech } \\
\text { Republic: Did They Matter? }\end{array}$ & Balázs Égert and Luboš Komárek & Mar. 2005 \\
\hline $\begin{array}{l}\text { No. 759: Assessing Market Expectations on Exchange Rates and } \\
\text { Inflation: A Pilot Forecasting System for Bulgaria }\end{array}$ & $\begin{array}{l}\text { Michael Berlemann, Kalina } \\
\text { Dimitrova, \& Nikolay Nenovsky }\end{array}$ & Mar. 2005 \\
\hline No. 758: Attitudes and Performance: An Analysis of Russian Workers & $\begin{array}{l}\text { Susan J. Linz and Anastasia } \\
\text { Semykina }\end{array}$ & Mar. 2005 \\
\hline $\begin{array}{l}\text { No. 757: Barter, Credit, and Welfare: A theoretical inquiry into the } \\
\text { barter phenomenon in Russia }\end{array}$ & José Noguera and Susan J. Linz & Mar. 2005 \\
\hline $\begin{array}{l}\text { No. 756: Sorting, Selection, and Transformation of the Return to } \\
\text { College Education In China }\end{array}$ & $\begin{array}{l}\text { Belton M. Fleisher, Haizheng Li, } \\
\text { Shi Li, and Xiaojun Wang }\end{array}$ & Mar. 2005 \\
\hline $\begin{array}{l}\text { No. 755: Foreign Exchange Interventions in Emerging Europe: } \\
\text { Should We Give a Damn? The Case of Croatia and Turkey }\end{array}$ & Balázs Égert and Maroje Lang & Mar. 2005 \\
\hline $\begin{array}{l}\text { No. 754: Targeting Relative Inflation Forecast as Monetary Policy } \\
\text { Framework for Adopting Euro }\end{array}$ & Lucjan T. Orlowski & Feb. 2005 \\
\hline $\begin{array}{l}\text { No. 753: Internet Entrepreneurship: Networks and Performance of } \\
\text { Internet Ventures In China }\end{array}$ & Bat Batjargal & Feb. 2005 \\
\hline $\begin{array}{l}\text { No. 752: Network Triads: Transitivity, Referral and Venture Capital } \\
\text { Decisions in China and Russia }\end{array}$ & Bat Batjargal & Feb. 2005 \\
\hline $\begin{array}{l}\text { No. 751: Software Entrepreneurship: Knowledge Networks and } \\
\text { Performance Of Software Ventures In China and Russia }\end{array}$ & Bat Batjargal & Feb. 2005 \\
\hline $\begin{array}{l}\text { No. 750: Retained State Shareholding in Chinese PLCs: Does } \\
\text { Government Ownership Reduce Corporate Value? }\end{array}$ & Lihui Tian and Saul Estrin & Feb. 2005 \\
\hline No. 749: Financial Development and Technology & Solomon Tadesse & Feb. 2005 \\
\hline No. 748: Banking Fragility and Disclosure: International Evidence & Solomon Tadesse & Feb. 2005 \\
\hline $\begin{array}{l}\text { No. 747: Consolidation, Scale Economies and Technological Change in } \\
\text { Japanese Banking }\end{array}$ & Solomon Tadesse & Feb. 2005 \\
\hline $\begin{array}{l}\text { No. 746: Trade Creation and Diversion Effects of Europe’s Regional } \\
\text { Liberalization Agreements }\end{array}$ & Yener Kandogan & Feb. 2005 \\
\hline No. 745: Quality of Institutions, Credit Markets and Bankruptcy & Christa Hainz & Feb. 2005 \\
\hline $\begin{array}{l}\text { No. 744: How Transition Paths Differ: Enterprise Performance in Russia } \\
\text { and China }\end{array}$ & Sumon Bhaumik and Saul Estrin & Jan. 2005 \\
\hline $\begin{array}{l}\text { No. 743: Inflation Targeting, Between Rhetoric and Reality. The Case } \\
\text { of Transition Economies }\end{array}$ & Daniel Daianu and Laurian Lungu & Jan. 2005 \\
\hline $\begin{array}{l}\text { No. 742: How Does Law Affect Finance? An Empirical Examination of } \\
\text { Tunneling in an Emerging Market }\end{array}$ & $\begin{array}{l}\text { Vladimir Atanasov, Conrad S. } \\
\text { Ciccotello, \& Stanley B. Gyoshev }\end{array}$ & Jan. 2005 \\
\hline $\begin{array}{l}\text { No. 741: Do Insider Trading Laws Matter? Some Preliminary } \\
\text { Comparative Evidence }\end{array}$ & Laura Nyantung Beny & Jan. 2005 \\
\hline $\begin{array}{l}\text { No. 740: Autopsy on an Empire: Understanding Mortality in Russia and } \\
\text { the Former Soviet Union }\end{array}$ & $\begin{array}{l}\text { Elizabeth Brainerd and David M. } \\
\text { Cutler }\end{array}$ & Jan. 2005 \\
\hline $\begin{array}{l}\text { No. 739: Not Separate, Not Equal: Poverty and Inequality in Post- } \\
\text { Apartheid South Africa }\end{array}$ & $\begin{array}{l}\text { Johannes G. Hoogeveen and Berk } \\
\text { Özler }\end{array}$ & Jan. 2005 \\
\hline $\begin{array}{l}\text { No. 738: The Marketing Structure in Agribusiness during the Transition } \\
\text { in Bulgaria }\end{array}$ & $\begin{array}{l}\text { Steve Murray, Yordan Staykov, } \\
\text { and Valentin Katzerov }\end{array}$ & Jan. 2005 \\
\hline No. 737: Passive Creditors & $\begin{array}{l}\text { Koen Schoors and Konstantin } \\
\text { Sonin }\end{array}$ & Jan. 2005 \\
\hline
\end{tabular}

